\title{
LOCAL ESTIMATES FOR PARABOLIC EQUATIONS WITH NONLINEAR GRADIENT TERMS
}

\author{
TOMMASO LEONORI AND FRANCESCO PETITTA
}

\begin{abstract}
In this paper we deal with local estimates for parabolic problems in $\mathbb{R}^{N}$ with absorbing first order terms, whose model is

$$
\begin{cases}u_{t}-\Delta u+u|\nabla u|^{q}=f(t, x) & \text { in }(0, T) \times \mathbb{R}^{N}, \\ u(0, x)=u_{0}(x) & \text { in } \mathbb{R}^{N},\end{cases}
$$

where $T>0, N \geq 2,1<q \leq 2, f(t, x) \in L^{1}\left(0, T ; L_{\text {loc }}^{1}\left(\mathbb{R}^{N}\right)\right)$ and $u_{0} \in$ $L_{\text {loc }}^{1}\left(\mathbb{R}^{N}\right)$.
\end{abstract}

\section{INTRODUCTION}

In this paper we deal with local estimates for parabolic problems in $\mathbb{R}^{N}, N \geq 2$, with absorbing first order lower order terms. In particular, our main goal concerns the proof of the existence of a solution for Cauchy problems whose model is

$$
\begin{cases}u_{t}-\Delta_{p} u+u|\nabla u|^{q}=f(t, x) & \text { in }(0, T) \times \mathbb{R}^{N}, \\ u(0, x)=u_{0}(x) & \text { in } \mathbb{R}^{N}\end{cases}
$$

where $T>0, p>1, p-1<q \leq p, f(t, x) \in L^{1}\left(0, T ; L_{\text {loc }}^{1}\left(\mathbb{R}^{N}\right)\right)$ and $u_{0} \in$ $L_{\text {loc }}^{1}\left(\mathbb{R}^{N}\right)$, without any prescribed behavior of the solutions at infinity. Here $\Delta_{p} u \equiv$ $\operatorname{div}\left(|\nabla u|^{p-2} \nabla u\right)$ is the usual $p$-laplace operator.

Such a problem is obviously strictly related to the possibility of proving estimates for the solutions that are independent of their behavior at infinity; this is a peculiarity of nonlinear equations with strong absorption lower order terms. For instance, if we consider the heat equation in the whole space $\mathbb{R}^{N}$, it is well known that the solution is explicit and turns out to be the convolution of the data with the heat kernel. Thus it is clear that, roughly speaking, $\forall(t, x) \in(0, T) \times \mathbb{R}^{N}$, any $a$ priori estimates on the solution also depend on what happens far away from $(t, x)$, which means that local estimates do not, in general, hold true.

Therefore, the presence of the the absorption lower order term, and its regularizing effect, is crucial in order to prove local estimates; actually it plays the role of a barrier.

1991 Mathematics Subject Classification. 35K60 and 35D05 and 35D10.

Key words and phrases. Local estimates, Nonlinear parabololic equations and Entire solutions.

The first author has been partially supported by MICINN Ministerio de Ciencia e Innovación (Spain) MTM2009-10878 and Junta de Andalucía FQM-116.

The second author has been partially supported by the PNPGC project, references MTM200803176. 
If such a term does not depend on the gradient, i.e. for problems of the type

$$
\begin{cases}u_{t}-\Delta_{p} u+b(u)=f(t, x) & \text { in }(0, T) \times \mathbb{R}^{N} \\ u(0, x)=u_{0}(x) & \text { in } \mathbb{R}^{N},\end{cases}
$$

with $f(t, x) \in L^{1}\left(0, T ; L_{\text {loc }}^{1}\left(\mathbb{R}^{N}\right)\right), b(\cdot) \in C^{0}(\mathbb{R})$, and $u_{0} \in L_{\text {loc }}^{1}\left(\mathbb{R}^{N}\right)$, the existence (and regularity) of distributional solutions has been investigated in 8 , and more recently in [21].

The main assumptions on the nonlinearity $b(u)$ are a sign condition (namely $b(s) s \geq 0, \forall s \in \mathbb{R})$ and an hypothesis on the behavior at infinity:

$$
\int^{\infty} \frac{d s}{(b(s) s)^{\frac{1}{p}}}<\infty .
$$

We recall that if $p=2$ (and if $b$ is increasing at least at infinity) (1.3) is equivalent to the well-known Keller-Osserman condition. Such a condition has been introduced in the pioneering papers 18 and 25] in order to prove a local (uniform) bound for any subsolution of the nonlinear elliptic equation

$$
-\Delta u+b(u)=f \quad \text { in } \Omega,
$$

where $\Omega \subset \mathbb{R}^{N}$ is bounded and $f \in L^{\infty}(\Omega)$. This tool is strictly related to the possibility of constructing solutions that blow-up at the boundary (the so called large solutions); since the literature on this topic is huge, we only mention, among the others, 3 and [30. We want to stress that (1.3) (as well as the Keller-Osserman condition) is the necessary and sufficient condition to have a global solution for the ordinary differential equation associated to the (elliptic) equation, namely

$$
\left\{\begin{array}{l}
\left(\left|v^{\prime}\right|^{p-2} v^{\prime}\right)^{\prime}=b(v) \quad \text { in }(0, \infty) \\
v(0)=+\infty
\end{array}\right.
$$

As we have already mentioned, local estimates are crucial in the study of large solutions for (1.4) and such a problem turns out to be strictly related to the study of the same problem in the whole $\mathbb{R}^{N}$ without conditions at infinity (see [11, [7] and 20 ).

On the other hand, nonlinear equations with absorption gradient terms have been studied since many years. It has been recently investigated (see 22]) the problem of both existence of solutions in the whole space (entire solutions) and existence of large solutions associated to nonlinear elliptic equations whose model is

$$
-\Delta_{p} u+u+u|\nabla u|^{q}=f(x) \text { in } \Omega,
$$

where $\Omega \subseteq \mathbb{R}^{N}, p>1, p-1<q \leq p$ and $f(x)$ is a singular datum (say $L^{1}(\Omega)$ ).

The purpose of this paper is twofold: on one side we want to extend the results of 21 to nonlinear problems with lower order terms that depend also on the gradient, namely of the type

$$
u_{t}-\Delta_{p} u+u|\nabla u|^{q}=f(t, x) \text { in }(0, T) \times \Omega,
$$

for $\Omega=\mathbb{R}^{N}$, equipped with an initial datum $u_{0}(x) \in L_{\text {loc }}^{1}(\Omega)$ and without any prescribed behavior at infinity for solutions. On the other hand, since we deal with local estimates, our aim is to show that we can construct solutions that assume, in a suitable sense, the value " $+\infty$ " at $(0, T) \times \partial \Omega$, if $\Omega$ is a bounded domain in $\mathbb{R}^{N}$. 
In order to prove such kind of results, we have to face several difficulties: first of all we have to give a consistent definition of solution. In this framework several notions of solutions have been considered and, according with the definition introduced in 5 for parabolic equations with $L^{1}$ data (see also [15], 14] and 26] for further generalizations to measure data), we use a renormalized formulation.

In fact, such a notion of solution turns out to be stronger than the distributional one, and it is very useful in order to face problems that involve singular data. Indeed, the peculiarity of such solutions is that they do not have local finite energy, but a priori estimates show that their truncations belong to the energy space; the idea is then to focus the attention to a family of problems solved by such truncations (see Definition 2.2 below).

The strategy we use in order to prove the existence results relies on the combination of both local estimates and local compactness results in suitable Sobolev spaces for solutions of certain approximating problems. It is clear that, since the formulation will involve cut-off functions, it will be independent of the behavior at infinity, if we are interested in the problem in the whole space. We also deal with the existence of large solutions for (1.5) in the case of $\Omega$ being a bounded domain. As far as large solutions are concerned, we have the additional problem of defining the explosive condition on $\partial \Omega \times(0, T)$ : since, a comparison principle does not hold true, we can not approach it by using a sub and supersolutions method. Moreover, the renormalized solutions are not, in general, continuous, so we have to define how the value $+\infty$ is achieved on the boundary in a convenient way. For this purpose we extend the definition given in 22] to the parabolic framework; roughly speaking, we say that a solution attains the value $+\infty$ on the boundary if its truncation at level $k$ has constant trace $k$ on the boundary, for any $k>0$. Because of this fact, in such a case, the truncations of the solutions have also to satisfy a global energy estimate in the whole $(0, T) \times \Omega$.

\section{Assumptions and statement of the main Results}

Let $\Omega$ be an open subset of $\mathbb{R}^{N}, N \geq 2$, possibly $\mathbb{R}^{N}$ itself, and let $T>0$. Throughout the paper we will use the following notation: for any ball $B_{r}$ of radius $r>0$ and $\forall \tau \in(0, T], Q_{r}^{\tau}=(0, \tau) \times B_{r}$, while $Q_{\Omega}^{\tau}=(0, \tau) \times \Omega$.

We consider the following equation

$$
u_{t}-\operatorname{div} a(t, x, u, \nabla u)+g(t, x, u, \nabla u)=f(t, x) \quad \text { in } Q_{\Omega}^{T},
$$

where $f(t, x) \in L^{1}\left(0, T ; L_{\text {loc }}^{1}(\Omega)\right)$ while $a(t, x, s, \varsigma):[0, T] \times \Omega \times \mathbb{R} \times \mathbb{R}^{N} \rightarrow \mathbb{R}^{N}$ is a Carathéodory function such that:

$$
\begin{array}{ll}
\exists \alpha>0: & a(t, x, s, \varsigma) \cdot \varsigma \geq \alpha|\varsigma|^{p}, \\
\exists \beta>0: & |a(t, x, s, \varsigma)| \leq \beta|\varsigma|^{p-1},
\end{array}
$$

and

$$
(a(t, x, s, \varsigma)-a(t, x, s, \eta)) \cdot(\varsigma-\eta)>0,
$$

for a.e. $(t, x) \in Q_{\Omega}^{T}, \forall s \in \mathbb{R}$, and $\forall \varsigma, \eta \in \mathbb{R}^{N}$ such that $\eta \neq \varsigma$ and $p>1$.

Under the above assumptions $\operatorname{div} a(t, x, u, \nabla u)$ turns out to be a Leray-Lions type operator, acting from $L^{p}\left(0, T ; W_{0}^{1, p}(\Omega)\right)$ into its dual (see [23]). 
As far as the lower order term is concerned we suppose that $g(t, x, s, \varsigma):[0, T] \times$ $\Omega \times \mathbb{R} \times \mathbb{R}^{N} \rightarrow \mathbb{R}$ is a Carathéodory function that satisfies the following; $\exists L>0$ such that

$$
\begin{gathered}
g(t, x, s, \varsigma) s \geq 0, \forall|s| \geq L, \forall \varsigma \in \mathbb{R}^{N}, \text { for a.e. }(t, x) \in Q_{\Omega}^{T}, \\
\forall k>0, \sup _{|s| \leq k}|g(t, x, s, \varsigma)| \leq\left|g_{k}\right|+\gamma_{k}|\varsigma|^{p}, \forall \varsigma \in \mathbb{R}^{N} \text {, for a.e. }(t, x) \in Q_{\Omega}^{T}, \\
\gamma_{k}>0, \quad g_{k}(t, x) \in L^{1}\left(0, T ; L_{\text {loc }}^{1}\left(\mathbb{R}^{N}\right)\right),
\end{gathered}
$$

and

$$
|g(t, x, s, \varsigma)| \geq h\left(|\varsigma|^{p-1}\right), \forall|s| \geq L, \forall \varsigma \in \mathbb{R}^{N}, \text { for a.e. }(t, x) \in Q_{\Omega}^{T},
$$

where $h$ is a positive $C^{2}\left(\mathbb{R}^{+}\right)$convex function $h$ such that $h(0)=0$, and the following conditions at infinity are satisfied:

$$
\int^{\infty} \frac{d \tau}{h(\tau)}<\infty \quad \text { and } \quad \limsup _{\tau \rightarrow \infty} \frac{\tau^{2} h^{\prime \prime}(\tau)}{h^{\prime}(\tau) \tau-h(\tau)}<\infty
$$

Some comments about these assumptions are in order to be given. Note that the absorption nature of the nonlinear lower order term depends on the sign condition (2.5), while (2.6) is known, in literature, as natural growth condition. We observe that condition (2.7) is a growth bound from below for the lower order term with respect to $\varsigma$ at infinity. This assumption is crucial, as it can be noticed in the proof of our main results, and, in particular, it plays a fundamental role in the construction of suitable cut-off functions needed to prove the local estimates we are interested in.

We remark that the first condition in (2.8) corresponds to the already mentioned Keller-Osserman assumption for equation (1.4). In fact, in the same spirit of the stationary case, it has to be imposed in order to prove the existence of a solution for the ordinary differential equation associated to (2.1). For instance, a solution for the problem

$$
\left\{\begin{array}{l}
\left(\left|v^{\prime}(s)\right|^{p-2} v^{\prime}(s)\right)^{\prime}=h\left(\left|v^{\prime}(s)\right|^{p-1}\right) \quad \text { in }(0,+\infty), \\
\lim _{s \rightarrow 0^{+}} v(s)=+\infty,
\end{array}\right.
$$

is well defined if and only if the first assumption in (2.8) holds true. Finally, the second assumption in (2.8) is technical and we expect that it could be removed.

Let us recall the standard notation for truncations, i.e. $T_{k}(s)=\max \{-k \min \{k, s\}\}$. We will, in general, handle with measurable functions whose truncations (locally) belong to the energy space $L^{p}\left(0, T ; W_{\text {loc }}^{1, p}\left(\mathbb{R}^{N}\right)\right)$. To do that it is useful to recall the notion of generalized gradient whose main feature is contained in the following result (see the proof in [4], Lemma 2.1).

Lemma 2.1. Let $\Omega \subseteq \mathbb{R}^{N}, N \geq 2$, and let $w(t, x)$ be a measurable a.e. finite function such that $T_{k}(w) \in L^{1}\left(0, T ; W_{\mathrm{loc}}^{1,1}(\Omega)\right), \forall k>0$. Then there exists a measurable function $v: Q_{\Omega}^{T} \mapsto \mathbb{R}^{N}$ such that $\forall k>0$ and for a.e. $(t, x) \in Q_{\Omega}^{T}$,

$$
\nabla T_{k}(w)=v \chi_{\{|w| \leq k\}} .
$$

Even if not explicitly stated, we will made use of this notion throughout the paper. Anyway, we recall that, if $w \in L^{1}\left(0, T ; W_{\mathrm{loc}}^{1,1}(\Omega)\right)$, then the generalized gradient coincides with the classical distributional one. 
Let us introduce the following definition of renormalized solution which is the natural extension of the classical one (see [5], [15] and [26]).

Definition 2.2. We say that a measurable function $u(t, x) \in L^{\infty}\left(0, T ; L_{\mathrm{loc}}^{1}(\Omega)\right)$ such that $\forall k>0, T_{k}(u) \in L^{p}\left(0, T ; W_{\text {loc }}^{1, p}(\Omega)\right)$ is a renormalized solution for equation (2.1), if $a(t, x, u, \nabla u) \in\left(L^{1}\left(0, T ; L_{\text {loc }}^{1}(\Omega)\right)\right)^{N}$, both $f(t, x)$ and $g(t, x, u, \nabla u)$ belong to $L^{1}\left(0, T ; L_{\mathrm{loc}}^{1}(\Omega)\right)$, and the following identity holds true:

$$
\begin{gathered}
-\int_{\Omega} S\left(u_{0}\right) \psi(x, 0)-\int_{0}^{T}\left\langle S(u), \psi_{t}\right\rangle \\
+\int_{Q_{\Omega}^{T}} a(t, x, u, \nabla u) \cdot \nabla u S^{\prime \prime}(u) \psi+\int_{Q_{\Omega}^{T}} a(t, x, u, \nabla u) \cdot \nabla \psi S^{\prime}(u) \\
+\int_{Q_{\Omega}^{T}} g(t, x, u, \nabla u) S^{\prime}(u) \psi=\int_{Q_{\Omega}^{T}} f(t, x) S^{\prime}(u) \psi,
\end{gathered}
$$

$\forall \psi \in C_{0}^{1}([0, T) \times \Omega)$, and for any $S(\tau) \in W^{2, \infty}(\mathbb{R})$ such that $S^{\prime}(\tau)$ is compactly supported on $\mathbb{R}$. Moreover,

$$
\lim _{l \rightarrow+\infty} \int_{Q_{\Omega}^{T} \cap\{l \leq|u| \leq l+1\}} a(t, x, u, \nabla u) \cdot \nabla u \Psi=0, \quad \forall \Psi \in C_{0}^{0}([0, T) \times \Omega) .
$$

Note that the regularity required for the solution is such that any term in (2.10) makes sense. In fact the above definition is nothing but equation (2.1) formally multiplied by $S^{\prime}(u) \psi$ and integrated on the cylinder $Q_{\Omega}^{T}$. The fact that $S^{\prime}$ is compactly supported ensures that all but the first two terms in (2.10) involve only a truncature of $u$. Condition (2.11) is necessary to recover a uniform information on $u$ on the set where it is large.

Finally, some comments regarding the initial datum are in order to be given: a priori, we are not in the position to apply Theorem 1.1 in [27] in order to deduce that $u \in C^{0}\left([0, T] ; L_{\mathrm{loc}}^{1}(\Omega)\right)$, since we have not imposed any regularity on $u_{t}$. Anyway, this result can be applied to $S(u)$, for any $S$ as above, since, by the equation, the distributional time-derivative $S(u)_{t}$ turns out to belong to $L^{1}\left(0, T ; L^{1}(\omega)\right)+$ $L^{p^{\prime}}\left(0, T ; W^{-1, p^{\prime}}(\omega)\right)$, for any $\omega \subset \subset \Omega$. Actually, this is enough in order to give sense to the formulation, but does not imply any information about the continuity of $u$.

Here we state our existence result concerning entire solutions.

Theorem 2.3. Assume that $a(t, x, s, \varsigma)$ and $g(t, x, s, \varsigma)$ satisfy 2.2 2.4 and (2.8) - 2.7), respectively. Then for any $f \in L^{1}\left(0, T ; L_{\text {loc }}^{1}\left(\mathbb{R}^{N}\right)\right)$ and for any $u_{0} \in$ $L_{\text {loc }}^{1}\left(\mathbb{R}^{N}\right)$ there exists a renormalized solution $u$ of the Cauchy problem

$$
\begin{cases}u_{t}-\operatorname{div} a(t, x, u, \nabla u)+g(t, x, u, \nabla u)=f(t, x) & \text { in }(0, T) \times \mathbb{R}^{N} \\ u(0, x)=u_{0}(x) & \text { in } \mathbb{R}^{N} .\end{cases}
$$

Moreover $u \in C^{0}\left([0, T] ; L_{l o c}^{1}\left(\mathbb{R}^{N}\right)\right)$.

As a consequence of the local estimates proved in the previous result, we are able to show the existence of a large solution for the boundary value problem associated to equation (2.1). More precisely, let $\Omega \subset \mathbb{R}^{N}$ be a bounded open subset of $\mathbb{R}^{N}$, $N \geq 2$. We consider the following problem: 


$$
\begin{cases}u_{t}-\operatorname{div} a(t, x, u, \nabla u)+g(t, x, u, \nabla u)=f(t, x) & \text { in }(0, T) \times \Omega \\ u(t, x)=+\infty & \text { on } \partial_{\mathcal{P}} Q_{\Omega}^{T} \\ u(0, x)=u_{0}(x) & \text { in } \Omega .\end{cases}
$$

where $\partial_{\mathcal{P}} Q_{\Omega}^{T}$ denotes the parabolic vertical boundary $(0, T) \times \partial \Omega$.

In the sequel we will need a suitable version of (2.6) adapted for this context, namely

$$
\begin{aligned}
\forall k>0 & \sup _{|s| \leq k}|g(t, x, s, \varsigma)| \leq\left|g_{k}(t, x)\right|+\gamma_{k}|\varsigma|^{p}, \\
& \gamma_{k}>0, \quad g_{k}(t, x) \in L^{1}\left(Q_{\Omega}^{T}\right) .
\end{aligned}
$$

Let us also specialize the definition of renormalized solution to this particular boundary value problem.

To our knowledge large solutions for parabolic equations have been investigated, for semilinear equations, in 2 2 and [1]. However, for such class of equations, solutions are continuous, so that the explosive condition makes sense pointwise.

For our purpose, we need to reformulate this condition in a suitable weak sense adapted to our framework. More precisely, the value " $u=+\infty$ " at $\partial \Omega$ is assumed through a condition on the trace of $T_{k}(u)$.

Definition 2.4. Let $\Omega$ be a bounded open subset of $\mathbb{R}^{N}, N \geq 2$. For any $f(t, x) \in$ $L^{1}\left(0, T ; L_{\text {loc }}^{1}(\Omega)\right)$, we define a renormalized large solution for problem (2.13) to be a measurable function $u(t, x)$ such that $T_{k}(u) \in L^{p}\left(0, T ; W^{1, p}(\Omega)\right), a(t, x, u, \nabla u) \in$ $\left(L^{1}\left(0, T ; L_{\mathrm{loc}}^{1}(\Omega)\right)\right)^{N}, g\left(t, x, u_{n}, \nabla u_{n}\right) \in L^{1}\left(0, T ; L_{\mathrm{loc}}^{1}(\Omega)\right)$ and it satisfies both (2.10) and (2.11). Moreover the boundary condition is assumed in the following sense:

$$
k-T_{k}(u) \in L^{p}\left(0, T ; W_{0}^{1, p}(\Omega)\right) \quad \forall k>0 .
$$

Our result concerning the existence of a large solution is the following one.

Theorem 2.5. Assume that $a(t, x, s, \varsigma)$ and $g(t, x, s, \varsigma)$ satisfy (2.2)-2.4 and (2.5), 2.7), (2.8) and 2.14). Then for any $f \in L^{1}\left(0, T ; L_{\mathrm{loc}}^{1}(\Omega)\right)$ such that $f^{-} \in L^{1}\left(Q_{\Omega}^{T}\right)$ and for any $u_{0} \in L_{\text {loc }}^{1}(\Omega)$ such that $u_{0}^{-} \in L^{1}(\Omega)$ there exists a (renormalized) large solution $u \in C^{0}\left([0, T] ; L_{l o c}^{1}(\Omega)\right)$ of problem (2.13).

We are also interested in some regularity properties for the renormalized solutions of (2.1) both if $\Omega$ is bounded and if $\Omega=\mathbb{R}^{N}$. Thus, let us introduce, for any $0<q<\infty$, the Marcinkiewicz space $M^{q}\left(Q_{\Omega}^{T}\right)$ as the space of all measurable functions $f$ such that there exists $c>0$, with

$$
\operatorname{meas}\left\{(t, x) \in Q_{\Omega}^{T}:|f(t, x)| \geq k\right\} \leq \frac{c}{k^{q}},
$$

for every positive $k$ endowed with the seminorm

$$
\|f\|_{M^{q}\left(Q_{\Omega}^{T}\right)}=\inf \left\{c>0: \operatorname{meas}\{(t, x):|f(t, x)| \geq k\} \leq\left(\frac{c}{k}\right)^{q}\right\} .
$$

Let us recall that, if $\Omega$ is bounded, then for $q>1$ we have the following continuous embeddings

$$
L^{q}\left(Q_{\Omega}^{T}\right) \hookrightarrow M^{q}\left(Q_{\Omega}^{T}\right) \hookrightarrow L^{q-\varepsilon}\left(Q_{\Omega}^{T}\right),
$$

for every $\varepsilon \in(0, q-1]$. 
We stress that from the definition of renormalized solution we can not, a priori, deduce neither any summability properties nor that $u$ has some continuity property in time for $p$ small. However, the following result holds.

Proposition 2.6. Any renormalized solution of (2.1) with initial datum $u_{0} \in$ $L_{\text {loc }}^{1}(\Omega)$ satisfies the following estimates:

$$
\|u\|_{M_{\mathrm{loc}}^{p-1+\frac{p}{N}}\left(Q_{\Omega}^{T}\right)} \leq c_{1} \quad \text { and } \quad\|\nabla u\|_{M_{\mathrm{loc}}^{p-\frac{N}{N+1}}\left(Q_{\Omega}^{T}\right)} \leq c_{2},
$$

where $c_{1}$ and $c_{2}$ are positive constants only depending on $u_{0}, f, N, R, T$ and $p$. Moreover if $p>2-\frac{1}{N+1}$ then $u \in C^{0}\left([0, T] ; L_{\mathrm{loc}}^{1}(\Omega)\right)$.

As already mentioned, for $1<p \leq 2-\frac{1}{N+1}$ the continuity with values in $L_{\text {loc }}^{1}$ can not be deduced a priori by embedding theorems since the gradient does not in general belong to any Lebesgue space. However the definition of renormalized solutions we gave above is not affected since, in order to give sense to the formulation, it is only required to $S(u)$ to admit a trace at $t=0$. Nevertheless, as stated in Theorem 2.3, we shall see that the solution we have found satisfies this condition for any $p>1$.

We finally want to investigate how the local summability of the datum $f(t, x)$ influences the local summability of the renormalized solutions. In particular we will show that the regularity of the solutions is, locally, the same of the solutions of equation (2.1) with $g \equiv 0$ and equipped with homogeneous Dirichlet conditions at the vertical boundary.

The techniques we use are nowadays classic and follow, for instance [17] and [10. However, since a localization is needed, the role of the lower order term (and in particular the growth condition (2.8) ) is crucial. Actually we will only sketch the proof of such result, underlining the main differences with the cases treated both in [17] and in [10.

Theorem 2.7. Suppose $2-\frac{1}{N+1}<p<N, q>1, m>1$, and suppose that $f(t, x)$ belongs to $L^{m}\left(0, T ; L_{\mathrm{loc}}^{q}(\Omega)\right)$. Then for any renormalized solution of (2.1) there exists $C_{0}$ (depending on $u_{0}, f, N, \Omega$ and $T$ ) such that,

(i) if

$$
1<\frac{1}{m}+\frac{N}{p q} \leq 1+\frac{N}{p m}
$$

for any initial datum $u_{0} \in L_{\text {loc }}^{N q \frac{p-2+m^{\prime}}{N m^{\prime}-p q}}(\Omega)$, then

$$
\|u\|_{L^{s}\left(0, T ; L_{\mathrm{loc}}^{s}(\Omega)\right)} \leq C_{0}, \quad \text { where } s=\frac{m q(N+p)+N(p-2)(q(m-1)+m)}{m N-p q(m-1)} ;
$$

moreover

$$
\begin{gathered}
\|u\|_{L^{s_{1}}\left(0, T ; L_{\mathrm{loc}}^{s_{2}}(\Omega)\right)} \leq C_{0}, \quad \text { where } s_{1}=m^{\prime} s_{0}, s_{2}=q^{\prime} s_{0} \\
\text { and } s_{0}=\frac{m q(q-1)+q(m-1)[p(N+1)-2 N]}{m N-p q(m-1)} .
\end{gathered}
$$

(ii) If

$$
\frac{1}{m}+\frac{N}{p q}>1+\frac{N}{p m}
$$




$$
\begin{aligned}
& \text { for any initial datum } u_{0} \in L_{\mathrm{loc}}^{\frac{N(q-1)(p-1)+N-p q}{N-p q}}(\Omega) \text {, then } \\
& \|u\|_{L^{s}\left(0, T ; L_{\mathrm{loc}}^{s}(\Omega)\right)} \leq C_{0}, \quad \text { where } s=\frac{[N(p-1)(q-1)+N-p q](N+p)}{N(N-p q)}+p-2 \text {; } \\
& \text { moreover } \\
& \|u\|_{L^{s_{1}}\left(0, T ; L_{\mathrm{loc}}^{s_{2}}(\Omega)\right)} \leq C_{0} \text {, where } s_{1}=m^{\prime} s_{0}, s_{2}=q^{\prime} s_{0} \quad \text { and } s_{0}=\frac{N(q-1)(p-1)}{N-p q} \text {. }
\end{aligned}
$$

(iii) If

$$
\begin{gathered}
\qquad \frac{1}{m}+\frac{N}{p q}<1, \\
\text { for any initial datum } u_{0} \in L_{\mathrm{loc}}^{\infty}(\Omega) \text {, then }\|u\|_{L^{\infty}\left(0, T ; L_{\mathrm{loc}}^{\infty}(\Omega)\right)} \leq C_{0} .
\end{gathered}
$$

Let us only notice that, as a typical smoothing effect for parabolic problems, the summability of the solution in space (e.g. $s_{2}$ in Theorem 2.7) is always greater than the summability of the initial datum.

Notation. Define $\varphi_{\lambda}(s)=s e^{\lambda s^{2}}$; we recall that $\varphi_{\lambda}(s)$ enjoys the following useful property:

$$
\forall a>0, b>0, \forall \lambda>\frac{b^{2}}{8 a^{2}} \quad a \varphi_{\lambda}^{\prime}(s)-b\left|\varphi_{\lambda}(s)\right| \geq 1, \quad \forall s \in \mathbb{R} .
$$

We will also make use of the following functions related with the truncations:

$$
S_{j}(\tau)=\int_{0}^{\tau}\left[1-T_{1}\left(G_{j}(s)\right)\right] d s
$$

and $G_{k}(s)=s-T_{k}(s)$.

By $\langle\cdot, \cdot\rangle$ we mean the duality between suitable spaces in which function are involved. In particular we will consider both the duality between $W_{0}^{1, p}(\Omega)$ and $W^{-1, p^{\prime}}(\Omega)$ and the duality between $W_{0}^{1, p}(\Omega) \cap L^{\infty}(\Omega)$ and $W^{-1, p^{\prime}}(\Omega)+L^{1}(\Omega)$.

Finally, we use the following notation for sequences: $\varepsilon(\sigma, n, \nu)$ will indicate any quantity that vanishes as the parameters go to their (obvious, if not explicitly stressed) limit point, with the same order in which they appear, that is,

$$
\lim _{\nu \rightarrow \infty} \limsup _{n \rightarrow+\infty} \limsup _{\sigma \rightarrow \infty}|\varepsilon(\sigma, n, \nu)|=0 .
$$

We will also sometimes omit the dependence of $\varepsilon(\cdot)$ on one or more of its arguments, when they are not present.

\section{TEChNicAl RESUlts}

In this section we collect some technical results that will be useful in the rest of the paper. The first one concerns the construction of a suitable family of functions.

Proposition 3.1. Let $h: \mathbb{R}^{+} \rightarrow \mathbb{R}^{+}$be a $C^{2}$, convex function, such that $h(0)=0$, and such that (2.8) holds. Then, for any $\delta>0$, there exists a constant $C_{0}=C_{0}(\delta)>$ 0 and a function $\sigma:[0,1] \rightarrow[0,1], \sigma \in C^{0}([0,1]) \cap C^{1}((0,1))$ with $\sigma(0)=\sigma^{\prime}(0)=0$, $\sigma(1)=1$, such that

$$
\forall v>0, \quad v \sigma^{\prime}(s) \leq \delta h(v) \sigma(s)+C_{\delta}, \quad \forall s \in[0,1] .
$$


Before giving the proof of Proposition 3.1. we need to introduce another fundamental tool in our arguments, that is a generalized Young inequality with the function $h$ which appears in (2.7)-(2.8). In order to do that, we have to introduce the Legendre transform for $h$ together with its properties which we will use in the sequel.

We recall that $h$ is a $C^{2}$ increasing and convex function such that $h(0)=0$. Moreover by the convexity and since (2.8) holds (i.e., roughly speaking, $h$ is $a$ bit more than superlinear at infinity) it follows that

$$
\lim _{s \rightarrow \infty} h^{\prime}(s)=+\infty .
$$

Let us consider the Legendre transform of $h$ defined by

$$
h^{*}(q)=\sup _{r \in \mathbb{R}}[q r-h(r)] .
$$

Here we recall the so called generalized Young inequality; namely, for any positive $z, w$, we have

$$
w z \leq h(z)+h^{*}(w) .
$$

It is clear that $h^{*}$ is continuous, increasing and, since (3.2) holds, superlinear at infinity. Consequently $h^{*-1}$ is well defined and moreover

$$
\lim _{q \rightarrow \infty} h^{*-1}(q)=+\infty .
$$

Moreover, since $h$ is smooth, $\forall q>0$, we have

$$
h^{*}(q)=q\left[\left(h^{\prime}\right)^{-1}(q)\right]-h\left(\left(h^{\prime}\right)^{-1}(q)\right),
$$

so that

$$
h^{*}\left(h^{\prime}(y)\right)=y h^{\prime}(y)-h(y), \quad \forall y>0 .
$$

The proof of Proposition 3.1 is based on the possibility of constructing a solution of a suitable Cauchy problem, as stated in the following Lemma.

Lemma 3.2. Let $h: \mathbb{R}^{+} \rightarrow \mathbb{R}^{+}$be a $C^{2}$, convex function, such that $h(0)=0$, and such that (2.8) holds. Then, for any $\delta>0$ there exists $C_{0}=C_{0}(\delta)$ and a function $\sigma=\sigma_{\delta}:[0,1] \rightarrow[0,1], \sigma \in C^{0}([0,1]) \cap C^{1}((0,1))$ solution of the problem

$$
\left\{\begin{array}{l}
\sigma^{\prime}(s)=\delta \sigma(s) h^{*-1}\left(\frac{C_{0}}{\delta \sigma(s)}\right) \quad \text { in }(0,1), \\
\sigma(0)=0, \quad \sigma(s)>0 .
\end{array}\right.
$$

Moreover

$$
\sigma(1)=1, \quad \text { and } \quad \lim _{s \rightarrow 0^{+}} \sigma^{\prime}(s)=0 .
$$

Proof. Let us consider the family of functions $\sigma(s)$ defined by the implicit formula

$$
\int_{0}^{\sigma(s)} \frac{d t}{\left(h^{*}\right)^{-1}\left(\frac{\tau}{t}\right) t}=s \delta, \quad \forall \tau>0 .
$$

Our aim is to prove that $\sigma$ is well defined and that there exists a value $\tau=C_{0}$ such that (3.3) and (3.4) hold true.

Step 1: Near 0. We want to prove that $\forall \tau>0, \sigma(s)$ is well defined in a neighborhood of $s=0$. Indeed, through the change of variable defined by the 
relationship $h^{\prime}(z)=\left(h^{*}\right)^{-1}\left(\frac{\tau}{\delta \sigma(s)}\right)$, and by the properties of $h$ and $h^{*}$ we have stated before, it follows that

$$
\frac{1}{\delta} \int_{0} \frac{d t}{\left(h^{*}\right)^{-1}\left(\frac{\tau}{t}\right) t}<+\infty \Leftrightarrow \frac{1}{\delta} \int^{+\infty} \frac{z h^{\prime \prime}(z)}{h^{\prime}(z)\left[h^{\prime}(z) z-h(z)\right]} d z<+\infty .
$$

Recalling (2.8) and since $h^{\prime}(z) z-h(z)>0$, for any $z>0$, there exists a constant $c_{1}$ such that

$$
\int^{+\infty} \frac{1}{z h^{\prime}(z)} \frac{z^{2} h^{\prime \prime}(z)}{h^{\prime}(z) z-h(z)} d z \leq c_{1} \int^{+\infty} \frac{d z}{h^{\prime}(z) z} d z \leq c_{1} \int^{+\infty} \frac{d z}{h(z)},
$$

where the last inequality holds since $h$ is convex and $h(0)=0$ : by (2.8) the last integral is finite and so $\sigma$ is well defined near 0 .

Step 2: The choice of $C_{0}$. It follows by Step 1, through the change $\rho=\frac{\tau}{\delta t}$, $\forall \delta>0$, that

$$
\lim _{\tau \rightarrow+\infty} \frac{1}{\delta} \int_{\frac{\tau}{\delta}}^{\infty} \frac{d \rho}{\rho\left(h^{*}\right)^{-1}(\rho)}=0
$$

on the other hand, since $\left(h^{*}\right)^{-1}(0)=0$,

$$
\lim _{\tau \rightarrow 0} \frac{1}{\delta} \int_{\frac{\tau}{\delta}}^{\infty} \frac{d \rho}{\rho\left(h^{*}\right)^{-1}(\rho)}=+\infty
$$

Thus there exists $C_{0}$ such that

$$
\frac{1}{\delta} \int_{\frac{C_{0}}{\delta}}^{\infty} \frac{d \rho}{\rho\left(h^{*}\right)^{-1}(\rho)}=\int_{0}^{1} \frac{d t}{\left(h^{*}\right)^{-1}\left(\frac{C_{0}}{t}\right) t}=1,
$$

which implies $\sigma(1)=1$.

Step 3: The limit of $\sigma^{\prime}$. Recalling the definition of $\sigma^{\prime}$ from (3.3), we want to prove

$$
\lim _{s \rightarrow 0^{+}} \delta \sigma(s) h^{*-1}\left(\frac{C_{0}}{\delta \sigma(s)}\right)=0 .
$$

This is equivalent to prove that

$$
\lim _{\tau \rightarrow+\infty} \frac{h^{\prime}(\tau)}{h^{*}\left(h^{\prime}(\tau)\right)}=\lim _{\tau \rightarrow+\infty} \frac{h^{\prime}(\tau)}{h^{\prime}(\tau) \tau-h(\tau)}=0
$$

since $\tau$ is such that $\left(h^{*}\right)^{-1}\left(\frac{C_{0}}{\delta \sigma(s)}\right)=h^{\prime}(\tau)$. Using that $h^{*}\left(h^{\prime}(\tau)\right) \rightarrow+\infty$ as $\tau$ diverges and by De l'Hopital rule we deduce that (3.6) holds, and so the Lemma is proved.

of Proposition 3.1. Let $\sigma(s)$ be the function defined in Lemma 3.2, Thus it is clear that inequality (3.1) is satisfied at $s=0$, and we can multiply and divide the left hand side by $\sigma(s)$; using (3.2) we get

$$
\delta \sigma(s) v \frac{\sigma^{\prime}(s)}{\delta \sigma(s)} \leq \delta \sigma(s) h(v)+\delta \sigma(s) h^{*}\left(\frac{\sigma^{\prime}(s)}{\delta \sigma(s)}\right) .
$$

Recalling that $\sigma$ is the solution of the Cauchy problem defined in (3.3), (3.1) holds true. 
In the sequel we will also handle with dualities involving the time derivatives of suitable functions; to this aim we will use the following Landes-type (see [19]) regularization result.

Lemma 3.3. Let $\Omega$ be an open bounded subset of $\mathbb{R}^{N}$, and let $w \in L^{p}\left(0, T ; W_{0}^{1, p}(\Omega)\right)$ and $w_{0} \in L^{1}(\Omega)$. Then, for any $\nu>0$, there exists a function $\eta_{\nu}=\eta_{\nu}\left(w, w_{0}\right) \in$ $L^{p}\left(0, T ; W_{0}^{1, p}(\Omega)\right)$, such that

$$
\frac{d}{d t} \eta_{\nu}=\nu\left(w-\eta_{\nu}\right)
$$

and $\eta_{\nu}\left(w, w_{0}\right)(0, x)=\eta_{0, \nu} \in L^{2}(\Omega)$, with

$$
\eta_{0, \nu} \stackrel{\nu \rightarrow \infty}{\longrightarrow} w_{0} \text { in } L^{1}(\Omega) .
$$

If furthermore $w \in L^{\infty}\left(Q_{\Omega}^{T}\right)$, then

$$
\left\|\eta_{\nu}\right\|_{L^{\infty}(Q)} \leq\|w\|_{L^{\infty}\left(Q_{\Omega}^{T}\right)} .
$$

Moreover, if $w_{t}=w^{(1)}+w^{(2)} \in L^{1}\left(Q_{\Omega}^{T}\right)+L^{p^{\prime}}\left(0, T ; W^{-1, p^{\prime}}(\Omega)\right)$, then $\frac{d}{d t} \eta_{\nu}$ admits a decomposition of the form $\frac{d}{d t} \eta_{\nu}=\rho_{\nu}^{(1)}+\rho_{\nu}^{(2)}$, with both

$$
\rho_{\nu}^{(1)} \stackrel{\nu \rightarrow \infty}{\longrightarrow} w^{(1)} \text { in } L^{1}\left(Q_{\Omega}^{T}\right)
$$

and

$$
\rho_{\nu}^{(2)} \stackrel{\nu \rightarrow \infty}{\longrightarrow} w^{(2)} \quad \text { in } \quad L^{p^{\prime}}\left(0, T ; W^{-1, p^{\prime}}(\Omega)\right) .
$$

Proof. See [16, Lemma 2.1.

Here we state a useful result which allows us to handle functions that do not have time derivatives belonging to the dual of the energy space $L^{p}\left(0, T ; W_{0}^{1, p}(\Omega)\right)$. In fact it consists in a generalized integration by parts formula, whose proof can be found in [14] (see also [12]).

Lemma 3.4. Let $\Omega$ be any domain in $\mathbb{R}^{N}, N \geq 2$, and let $\phi: \mathbb{R} \rightarrow \mathbb{R}$ be a continuous piecewise $C^{1}$ function such that $\phi(0)=0$ and $\phi^{\prime}$ has compact support; let us define $\Phi(s)=\int_{0}^{s} \phi(r) d r$. If $v \in L^{p}\left(0, T, W_{0}^{1, p}(\Omega)\right)$ is such that $v_{t} \in$ $L^{p^{\prime}}\left(0, T ; W^{-1, p^{\prime}}(\Omega)\right)+L^{1}\left(Q_{\Omega}^{T}\right)$ and if $\psi \in C^{\infty}\left(\overline{Q_{\Omega}^{T}}\right)$, then we have

$$
\int_{0}^{T}\left\langle v_{t}, \phi(v) \psi\right\rangle=\int_{\Omega} \Phi(v(T)) \psi(T)-\int_{\Omega} \Phi(v(0)) \psi(0)-\int_{Q_{\Omega}^{T}} \psi_{t} \Phi(v) .
$$

We observe that $v_{t} \in L^{p^{\prime}}\left(0, T ; W^{-1, p^{\prime}}(\Omega)\right)+L^{1}\left(Q_{\Omega}^{T}\right)$ implies that there exist $\eta_{1} \in L^{p^{\prime}}\left(0, T ; W^{-1, p^{\prime}}(\Omega)\right)$ and $\eta_{2} \in L^{1}\left(Q_{\Omega}^{T}\right)$ such that $u_{t}=\eta_{1}+\eta_{2}$. Even if $\eta_{1}$ and $\eta_{2}$ are not uniquely determined, the integration by parts formula turns out to be independent of the representation of $v_{t}$; moreover, according with the notation introduced before, $\langle\cdot, \cdot\rangle$ will indicate the duality between $L^{p^{\prime}}\left(0, T ; W^{-1, p^{\prime}}(\Omega)\right)+$ $L^{1}\left(Q_{\Omega}^{T}\right)$ and $L^{p}\left(0, T ; W_{0}^{1, p}(\Omega)\right) \cap L^{\infty}\left(Q_{\Omega}^{T}\right)$.

We also recall the following classical result due to Gagliardo and Nirenberg.

Theorem 3.5 (Gagliardo-Nirenberg). Let $\Omega \subset \mathbb{R}^{N}$, open and bounded, and let $v$ be a function in $W^{1, \mu}(\Omega) \cap L^{\lambda}(\Omega)$ with $\mu \geq 1, \lambda \geq 1$. Then there exists a positive constant $C$, depending on $N, q$ and $\lambda$, such that

$$
\|v\|_{L^{\eta}(\Omega)} \leq C\|\nabla v\|_{\left(L^{\mu}(\Omega)\right)^{N}}^{\theta}\|v\|_{L^{\lambda}(\Omega)}^{1-\theta},
$$


for every $\theta$ and $\eta$ satisfying

$$
0 \leq \theta \leq 1, \quad 1 \leq \eta \leq+\infty, \quad \frac{1}{\eta}=\theta\left(\frac{1}{\mu}-\frac{1}{N}\right)+\frac{1-\theta}{\lambda} .
$$

Proof. See 24, Lecture II.

The following embedding results are consequences of the previous theorem. We will use them in the last section but we give here their statement for completeness.

Corollary 3.6. Let $v \in L^{q}\left(0, T ; W_{0}^{1, q}(\Omega)\right) \cap L^{\infty}\left(0, T ; L^{\gamma}(\Omega)\right)$, with $q \geq 1, \gamma \geq 1$. Then $v \in L^{\sigma}\left(Q_{\Omega}^{T}\right)$ with $\sigma=q \frac{N+\gamma}{N}$ and

$$
\int_{Q_{\Omega}^{T}}|v|^{\sigma} d x d t \leq C\|v\|_{L^{\infty}\left(0, T ; L^{\gamma}(\Omega)\right)}^{\frac{\gamma q}{N^{\infty}}} \int_{Q_{\Omega}^{T}}|\nabla v|^{q} d x d t .
$$

Corollary 3.7. Let $\Omega \subset \mathbb{R}^{N}$, open and bounded, $\tau>0,1<p<N$ and let further $w \in L^{\infty}\left(0, \tau ; L^{p}(\Omega)\right) \cap L^{p}\left(0, \tau ; W_{0}^{1, p}(\Omega)\right)$. Then there exists a positive constant $K$ depending only on $N$ and $p$ such that

$$
\left[\int_{0}^{\tau}\left(\int_{\Omega}|w|^{\sigma}\right)^{\frac{\mu}{\sigma}}\right]^{\frac{p}{\mu}} \leq K\left(\sup _{t \in[0, \tau]} \int_{\Omega}|w|^{p}+\int_{0}^{\tau} \int_{\Omega}|\nabla w|^{p}\right)
$$

for all $\mu$ and $\sigma$ satisfying

$$
p \leq \sigma \leq p^{*}, \quad p \leq \mu \leq \infty, \quad \frac{N}{p \sigma}+\frac{1}{\mu}=\frac{N}{p^{2}} .
$$

We also recall the interpolation inequality that we will use in the proof of Theorem 2.7 Assume that $z \in L^{\infty}\left(0, T ; L^{q}(\Omega)\right) \cap L^{p}\left(0, T ; L^{r}(\Omega)\right), p, q, r \geq 1$. Thus $z \in L^{\eta}\left(Q_{\Omega}^{T}\right)$ and

$$
\left\{\begin{array}{c}
\|z\|_{L^{\eta}(Q)} \leq C\|z\|_{L^{\infty}\left(0, T ; L^{q}(\Omega)\right)}^{1-\theta}\|z\|_{L^{p}\left(0, T ; L^{r}(\Omega)\right)}^{\theta} \\
\text { with } \quad \frac{1}{\eta}=\frac{\theta}{r}+\frac{1-\theta}{q}, p \geq \theta \eta
\end{array}\right.
$$

A useful application of Corollary 3.6 is the following.

Proposition 3.8. Let $\Omega \subset \mathbb{R}^{N}$ be a bounded domain and $p>1$. Let $w \in$ $L^{\infty}\left(0, T ; L^{1}(\Omega)\right)$ such that $T_{k}(w) \in L^{p}\left(0, T ; W_{0}^{1, p}(\Omega)\right)$, for any $k>0$. If $|\nabla w|^{p-1} \in$ $L^{1}\left(Q_{\Omega}^{T}\right)$, then $w^{p-1} \in L^{1}\left(Q_{\Omega}^{T}\right)$.

Proof. We deal only with the case $p>2$, since for $p \leq 2$ it is trivial. Since both $w \in L^{\infty}\left(0, T ; L^{1}(\Omega)\right)$ and $|\nabla w|^{p-1} \in L^{1}\left(Q_{\Omega}^{T}\right)$, we have that $w \in L^{1}\left(0, T ; W_{0}^{1,1}(\Omega)\right)$. Then, we can apply Corollary 3.6 with $q=\gamma=1$ to obtain that $w \in L^{\frac{N+1}{N}}\left(Q_{\Omega}^{T}\right)$. Now, if $p \leq 1+\frac{N+1}{N}$ we are finished, otherwise $w \in L^{1}\left(0, T ; W_{0}^{1, \frac{N+1}{N}}(\Omega)\right)$ and we apply again Corollary 3.6 with $\gamma=1$ and $q=\frac{N+1}{N}$ to conclude that $w \in$ $L^{\left(\frac{N+1}{N}\right)^{2}}\left(Q_{\Omega}^{T}\right)$. It is clear that, iterating this procedure, we get the result in a finite number of steps.

The estimates contained in the following lemma are standard and turn out to coincide, for instance, with the one proved in [6] (see also Lemma 3.7 in [21]). 
Lemma 3.9. Let $\Omega \subset \mathbb{R}^{N}$ be a bounded domain and let $w \in L^{p}\left(0, T ; W^{1, p}(\Omega)\right) \cap$ $L^{\infty}\left(0, T ; L^{1}(\Omega)\right), 1<p<N$. Suppose moreover that there exists $C_{0}>0$ depending only on $N, \Omega, T$ and $p$ such that

$$
\int_{Q_{\Omega}^{T}}\left|\nabla T_{k}(w)\right|^{p} \leq C_{0}(k+1) \quad \text { and } \quad \int_{0}^{T}|w| \leq C_{0}, \quad \forall k>0 .
$$

Then:

$$
\|w\|_{M^{s_{1}}\left(Q_{\Omega}^{T}\right)} \leq c_{1} \quad \text { and } \quad\|\nabla w\|_{M^{s_{2}}\left(Q_{\Omega}^{T}\right)} \leq c_{2}
$$

where

$$
\left\{\begin{array}{l}
s_{1}=\max \left\{1, p-1+\frac{p}{N}\right\} \\
s_{2}=\max \left\{\frac{p}{2}, p-\frac{N}{N+1}\right\},
\end{array}\right.
$$

and $c_{1}$ and $c_{2}$ are positive constants only depending on $C_{0}, N, \Omega, T$ and $p$.

Finally let us state the following classical result due to Stampacchia.

Lemma 3.10. Let $\zeta(j, \rho):[0,+\infty) \times[0, R)$ be a function such that $\zeta(\cdot, \rho)$ is nonincreasing and $\zeta(j, \cdot)$ nondecreasing. Moreover, suppose that $\exists K_{0}>0, \mu>1$, and $C, \nu, \gamma>0$ such that

$$
\zeta(j, \rho) \leq C \frac{\zeta(k, R)^{\mu}}{(j-k)^{\nu}(R-\rho)^{\gamma}} \quad \forall j>k>K_{0}, \forall \rho \in(0, R] .
$$

Then for every $\delta \in(0,1)$, there exists $d>0$ such that:

$$
\zeta\left(K_{0}+d,(1-\delta) R\right)=0
$$

where

$$
d^{\nu}=C^{\prime} 2^{\frac{\mu(\nu+\gamma)}{\mu-1}} \frac{\zeta\left(K_{0}, 1\right)^{\mu-1}}{(1-\delta)}, \quad C^{\prime}>0
$$

Proof. See [29].

\section{Proofs of Theorem 2.3 AND Theorem 2.5}

Here, and throughout the paper, we denote by $\xi=\xi_{R}^{\rho}(|x|)$ a $C_{0}^{1}\left(\mathbb{R}^{N}\right)$ function such that $\forall \rho>0$

$$
\begin{cases}\xi \equiv 1 & \text { if }|x| \leq R \\ 0<\xi<1 & \text { if } R<|x|<R+\rho \\ \xi \equiv 0 & \text { if }|x| \geq R+\rho .\end{cases}
$$

Note that this class of functions keeps its properties if composed with $C^{1}[0,1]$ functions $w:[0,1] \rightarrow[0,1]$ such that:

$$
w(0)=w^{\prime}(0)=0, \quad w(1)=1, \quad \text { and } \quad w(s)>0 \text { in }(0,1) .
$$

We will often choose a cut-off function $\xi=\sigma(\eta)$ where $\eta$ (and consequently $\xi$ ) satisfies (4.1) and $\sigma$ is the function that appears in Proposition 3.1, and thus such that (3.1) holds true for a suitable choice of $\delta$.

Now we can prove the first result concerning existence of solutions. 
of Theorem 2.3. . Let $B_{n}$ be the ball of radius $n$, centered at the origin (this choice can be done without loss of generality) and let $u_{n}$ be a weak solution of the following problem

$$
\begin{cases}\left(u_{n}\right)_{t}-\operatorname{div} a\left(t, x, u_{n}, \nabla u_{n}\right)+g\left(t, x, u_{n}, \nabla u_{n}\right)=f_{n}(t, x) & \text { in } Q_{n}^{T}, \\ u_{n}(t, x)=0 & \text { on } \partial_{\mathcal{P}} Q_{n}^{T}, \\ u_{n}(0, x)=u_{n}^{0}(x) & \text { in } B_{n},\end{cases}
$$

where $f_{n}(t, x)=T_{n}(f(t, x))$ and $u_{n}^{0}(x)=T_{n}\left(u_{0}(x)\right)$. Note that, thanks to the result of 13 (see also [27), there exists (at least) a weak solution for (4.2), i.e. a function $u_{n} \in L^{p}\left(0, T ; W_{0}^{1, p}\left(B_{n}\right)\right)$ such that $\left(u_{n}\right)_{t}$ belongs to $L^{p^{\prime}}\left(0, T ; W^{-1, p^{\prime}}\left(B_{n}\right)\right)$, $g\left(t, x, u_{n}, \nabla u_{n}\right)$ belongs to $L^{1}\left((0, T) \times B_{n}\right)$, and the following identity holds true

$$
\begin{gathered}
\int_{0}^{T}\left\langle\left(u_{n}\right)_{t}, \psi\right\rangle+\int_{Q_{n}^{T}} a\left(t, x, u_{n}, \nabla u_{n}\right) \cdot \nabla \psi \\
\quad+\int_{Q_{n}^{T}} g\left(t, x, u_{n}, \nabla u_{n}\right) \psi=\int_{Q_{n}^{T}} f_{n} \psi,
\end{gathered}
$$

$\forall \psi \in L^{p}\left(0, T ; W_{0}^{1, p}\left(B_{n}\right)\right) \cap L^{\infty}\left(Q_{n}^{T}\right)$.

We will prove Theorem 2.3 by showing that the terms in (4.2) are compact in suitable spaces. In order to do it, here and throughout the whole proof, we fix a ball $B_{R}$, centered at the origin, and we will prove suitable estimates for $u_{n}$ in $(0, T) \times B_{R}$. Moreover, a weak solution on $Q_{n}^{T}$ turns out to be obviously a weak solution in $Q_{n}^{t}$ for any $0<t<T$. Hence, with an abuse of notation, we will often refer to (4.3) by tacitly understanding its counterpart on $Q_{n}^{t}$.

Local estimates on truncations. For any $n \geq R+\rho$ (for any fixed $\rho>0$ ), let us choose in (4.3) $\psi=\varphi_{\lambda}\left(T_{k}\left(u_{n}\right)\right) \xi$, where $\varphi_{\lambda}(s)=s e^{\lambda s^{2}}$ ( $\lambda>0$ will be fixed later), $k>L$, and $\xi(x)$ is a cut-off function such that (4.1) holds true (we will often omit the dependence on $x$ ). Thus we have

$$
\begin{gathered}
\int_{0}^{t}\left\langle\left(u_{n}\right)_{t}, \varphi_{\lambda}\left(T_{k}\left(u_{n}\right)\right) \xi\right\rangle \\
+\int_{Q_{n}^{t}} a\left(t, x, u_{n}, \nabla u_{n}\right) \cdot \nabla T_{k}\left(u_{n}\right) \varphi_{\lambda}^{\prime}\left(T_{k}\left(u_{n}\right)\right) \xi \\
+\int_{Q_{n}^{t}} a\left(t, x, u_{n}, \nabla u_{n}\right) \cdot \nabla \xi \varphi_{\lambda}\left(T_{k}\left(u_{n}\right)\right) \\
+\int_{Q_{n}^{t}} g\left(t, x, u_{n}, \nabla u_{n}\right) \varphi_{\lambda}\left(T_{k}\left(u_{n}\right)\right) \xi=\int_{Q_{n}^{t}} f_{n}(t, x) \varphi_{\lambda}\left(T_{k}\left(u_{n}\right)\right) \xi .
\end{gathered}
$$

Since $\xi$ does not depend on time, using Lemma 3.4

$$
\int_{0}^{t}\left\langle\left(u_{n}\right)_{t}, \varphi_{\lambda}\left(T_{k}\left(u_{n}\right)\right) \xi\right\rangle=\int_{B_{n}} \Phi_{\lambda, k}\left(u_{n}(t, x)\right) \xi-\int_{B_{n}} \Phi_{\lambda, k}\left(u_{n}(x, 0)\right) \xi,
$$

where

$$
\Phi_{\lambda, k}(s)=\int_{0}^{s} \varphi_{\lambda}\left(T_{k}(\tau)\right) d \tau= \begin{cases}\frac{1}{2 \lambda}\left(e^{\lambda s^{2}}-1\right) & \text { if }|s| \leq k \\ \varphi_{\lambda}(k)(|s|-k)+\frac{1}{2 \lambda}\left(e^{\lambda k^{2}}-1\right) & \text { if }|s|>k\end{cases}
$$


Note that

$$
\varphi_{\lambda}(k)|s|-e^{\lambda k^{2}}\left(k^{2}-\frac{1}{2 \lambda}\right)-\frac{1}{2 \lambda} \leq \Phi_{\lambda, k}(s) \leq \varphi_{\lambda}(k)|s|,
$$

so that we deduce

$$
\begin{gathered}
\int_{0}^{t}\left\langle\left(u_{n}\right)_{t}, \varphi_{\lambda}\left(T_{k}\left(u_{n}\right)\right) \xi\right\rangle \\
\geq \varphi_{\lambda}(k) \int_{B_{n}}\left|u_{n}(t, x)\right| \xi-\left[e^{\lambda k^{2}}\left(k^{2}-\frac{1}{2 \lambda}\right)+\frac{1}{2 \lambda}\right] \operatorname{meas}\left\{B_{R+\rho}\right\} \\
-\varphi_{\lambda}(k) \int_{B_{n}}\left|u_{0}(x)\right| \xi
\end{gathered}
$$

We notice that assumptions (2.6)-(2.7) imply the following growth condition on $h$ :

$$
\exists c_{1}>0 \text { such that } h(\tau) \leq c_{1}\left(\tau^{\frac{p}{p-1}}+1\right), \forall \tau \in \mathbb{R}^{+} .
$$

Moreover, since $k>L$, by (2.5)-(2.8), we have

$$
\begin{gathered}
\int_{Q_{n}^{t}} g\left(t, x, u_{n}, \nabla u_{n}\right) \varphi_{\lambda}\left(T_{k}\left(u_{n}\right)\right) \xi \\
=\int_{Q_{n}^{t} \cap\left\{\left|u_{n}\right| \geq L\right\}} g\left(t, x, u_{n}, \nabla u_{n}\right) \varphi_{\lambda}\left(T_{k}\left(u_{n}\right)\right) \xi \\
+\int_{Q_{n}^{t} \cap\left\{\left|u_{n}\right| \leq L\right\}} g\left(t, x, u_{n}, \nabla u_{n}\right) \varphi_{\lambda}\left(T_{k}\left(u_{n}\right)\right) \xi \geq \int_{Q_{n}^{t}} h\left(\left|\nabla u_{n}\right|^{p-1}\right)\left|\varphi_{\lambda}\left(T_{k}\left(u_{n}\right)\right)\right| \xi \\
-\int_{Q_{n}^{t}}\left(\widetilde{\gamma}_{k}\left|\nabla T_{k}\left(u_{n}\right)\right|^{p}+\left|\widetilde{g_{k}}(t, x)\right|\right)\left|\varphi_{\lambda}\left(T_{k}\left(u_{n}\right)\right)\right| \xi,
\end{gathered}
$$

where $\widetilde{\gamma}_{k}=\gamma_{k}+c_{1}$ and $\widetilde{g}_{k}=g_{k}+c_{1}$, and $c_{1}$ is the constant appearing in (4.6). On the other hand by (2.3) and since $\xi$ can be chosen such that $\xi=\sigma(\eta)$, where $\eta$ satisfies (4.1), too, and $\sigma$ is the function defined in Lemma 3.2, we can apply Proposition 3.1 with $\delta=\frac{1}{2 \beta}$. Thus there exists a constant $C=C(\lambda, k, \beta, T)$ such that

$$
\begin{gathered}
\int_{Q_{n}^{t}} a\left(t, x, u_{n}, \nabla u_{n}\right) \cdot \nabla \xi \varphi_{\lambda}\left(T_{k}\left(u_{n}\right)\right) \\
\geq-\beta \int_{Q_{n}^{t}}\left|\nabla u_{n}\right|^{p-1}|\nabla \xi|\left|\varphi_{\lambda}\left(T_{k}\left(u_{n}\right)\right)\right| \\
\geq-\int_{Q_{n}^{t}} \frac{1}{2} h\left(\left|\nabla u_{n}\right|^{p-1}\right)\left|\varphi_{\lambda}\left(T_{k}\left(u_{n}\right)\right)\right| \xi-C \operatorname{meas}\left\{B_{R+\rho}\right\} .
\end{gathered}
$$

By substituting the above inequalities into (4.4), we deduce

$$
\begin{aligned}
& \varphi_{\lambda}(k) \int_{B_{n}}\left|u_{n}(t, x)\right| \xi+\int_{Q_{n}^{t}}\left|\nabla T_{k}\left(u_{n}\right)\right|^{p}\left[\alpha \varphi_{\lambda}^{\prime}\left(T_{k}\left(u_{n}\right)\right)-\widetilde{\gamma}_{k}\left|\varphi_{\lambda}\left(T_{k}\left(u_{n}\right)\right)\right|\right] \xi \\
& \leq \operatorname{meas}\left\{B_{R+\rho}\right\}+\varphi_{\lambda}(k)\left[\int_{B_{n}}\left|u_{0}(x)\right| \xi+\int_{Q_{n}^{t}}\left(\left|f_{n}(t, x)\right|+\left|\widetilde{g}_{k}(t, x)\right|\right) \xi\right] .
\end{aligned}
$$

Note that both $f_{n}(t, x) \xi$ and $\widetilde{g}_{k}(t, x) \xi$ are bounded in $L^{1}\left(Q_{R}^{T}\right)$, therefore, we choose $\lambda>\frac{\widetilde{\gamma}_{k}^{2}}{8 \alpha^{2}}$ so that (2.20) holds and we deduce that there exists a constant (depending on $k$ ) such that

$$
\sup _{t \in(0, T)} \int_{B_{R}}\left|u_{n}(t, x)\right|+\int_{Q_{R}^{T}}\left|\nabla T_{k}\left(u_{n}\right)\right|^{p} \leq C(k), \quad \forall k>0 .
$$


This implies, since obviously $\left\|T_{k}\left(u_{n}\right)\right\|_{L^{p}\left((0, T) \times B_{R}\right)} \leq C(R, T) k$, that $T_{k}\left(u_{n}\right)$ is bounded in $L^{p}\left(0, T ; W^{1, p}\left(B_{R}\right)\right), \forall R>0$. Thus, up to subsequences (not relabeled) $T_{k}\left(u_{n}\right)$ weakly converges toward a function $v_{k}$ in $L^{p}\left(0, T ; W^{1, p}\left(B_{R}\right)\right)$. Moreover the sequence $\left\{u_{n}\right\}$ is bounded in $L^{\infty}\left(0, T ; L^{1}\left(B_{R}\right)\right)$.

Hence, from (4.8) we deduce (integrating between 0 and $T$ ), $\forall j>0$,

$$
\begin{gathered}
j \operatorname{meas}\left\{(t, x) \in Q_{R}^{T}:\left|u_{n}\right| \geq j\right\} \\
\leq \int_{\left\{(0, T) \times B_{R+\rho}\right\} \cap\left\{(t, x):\left|u_{n}\right| \geq j\right\}}\left|u_{n}(t, x)\right| \xi \leq \int_{0}^{T} \int_{B_{n}}\left|u_{n}(t, x)\right| \xi \leq C T,
\end{gathered}
$$

so that

$$
\operatorname{meas}\left\{(t, x) \in Q_{R}^{T}:\left|u_{n}\right| \geq j\right\} \leq \frac{C T}{j} .
$$

Moreover, choosing $S_{k}^{\prime}\left(u_{n}\right) \xi$ as test function in (4.3) $\left(S_{k}\right.$ has been defined in (2.21) $)$, we deduce that $\left(S_{k}\left(u_{n}\right) \xi\right)$, is bounded in $L^{1}\left(Q_{R}^{T}\right)+L^{p^{\prime}}\left(0, T ; W^{-1, p^{\prime}}\left(B_{R+\rho}\right)\right)$ and so, using Corollary 4 in [28], we have that $S_{k}\left(u_{n}\right) \xi$ is strongly compact in $L^{1}\left((0, T) \times B_{R+\rho}\right)$. Hence, up to subsequences (not relabeled), it converges a.e. as $n$ diverges. Using a diagonal argument, it follows that $u_{n} \rightarrow u$ for a.e. $(t, x) \in Q_{R}^{T}$, $\forall R>0$, and consequently there exists a measurable function $u(t, x)$ such that $u_{n} \rightarrow u$ a.e. in $(0, T) \times \mathbb{R}^{N}$. Finally, we note that (4.9) and the a.e. convergence of $u_{n}$ imply, by Vitali's theorem, that $u_{n}$ is compact in $L^{1}\left(0, T ; L_{\text {loc }}^{1}\left(\mathbb{R}^{N}\right)\right)$ and consequently that

$$
T_{k}\left(u_{n}\right) \rightarrow T_{k}(u) \quad \text { in } L^{p}\left(0, T ; W_{\text {loc }}^{1, p}\left(\mathbb{R}^{N}\right)\right),
$$

so that $v_{k}=T_{k}(u)$.

Estimates on the lower order term. Let us choose, $\forall \varepsilon>0, \psi=\frac{T_{\varepsilon}\left(u_{n}\right)}{\varepsilon} \xi$ as test function in (4.3), so that we have:

$$
\begin{gathered}
\int_{Q_{n}^{T}} \frac{d}{d t}\left(\frac{\Theta_{\varepsilon}\left(u_{n}\right)}{\varepsilon}\right) \xi+\frac{1}{\varepsilon} \int_{Q_{n}^{T}} a\left(t, x, u_{n}, \nabla u_{n}\right) \cdot \nabla T_{\varepsilon}\left(u_{n}\right) \xi \\
+\int_{Q_{n}^{T}} a\left(t, x, u_{n}, \nabla u_{n}\right) \cdot \nabla \xi \frac{T_{\varepsilon}\left(u_{n}\right)}{\varepsilon} \\
+\int_{Q_{n}^{T}} g\left(t, x, u_{n}, \nabla u_{n}\right) \frac{T_{\varepsilon}\left(u_{n}\right)}{\varepsilon} \xi=\int_{Q_{n}^{T}} f_{n}(t, x) \frac{T_{\varepsilon}\left(u_{n}\right)}{\varepsilon} \xi \leq \int_{Q_{n}^{T}}\left|f_{n}(t, x)\right| \xi,
\end{gathered}
$$

where $\Theta_{k}(s)=\int_{0}^{s} T_{k}(\tau) d \tau, \forall k>0$. We first note that

$$
0 \leq \frac{\Theta_{\varepsilon}(s)}{\varepsilon} \leq|s|, \quad \forall s \in \mathbb{R},
$$

and by (2.2), we deduce

$$
\begin{gathered}
\frac{\alpha}{\varepsilon} \int_{Q_{n}^{T}}\left|\nabla T_{\varepsilon}\left(u_{n}\right)\right|^{p} \xi+\int_{Q_{n}^{T}} a\left(t, x, u_{n}, \nabla u_{n}\right) \cdot \nabla \xi \frac{T_{\varepsilon}\left(u_{n}\right)}{\varepsilon} \\
+\int_{Q_{n}^{T}} g\left(t, x, u_{n}, \nabla u_{n}\right) \frac{T_{\varepsilon}\left(u_{n}\right)}{\varepsilon} \xi \leq\left\|f_{n}(t, x)\right\|_{L^{1}\left(Q_{R+\rho}^{T}\right)}+\int_{B_{n}}\left|u_{n}^{0}(x)\right| \xi .
\end{gathered}
$$

Moreover, using (2.5)-(2.8), we have (as above $\widetilde{\gamma}_{L}=\gamma_{L}+c_{1}$ and $\widetilde{g}_{L}=g_{L}+c_{1}$ )

$$
\int_{Q_{n}^{T}} g\left(t, x, u_{n}, \nabla u_{n}\right) \frac{T_{\varepsilon}\left(u_{n}\right)}{\varepsilon} \xi
$$




$$
\begin{aligned}
& \geq \frac{1}{2} \int_{Q_{n}^{T}} h\left(\left|\nabla u_{n}\right|^{p-1}\right) \xi+\frac{1}{2} \int_{Q_{n}^{T}}\left|g\left(t, x, u_{n}, \nabla u_{n}\right)\right| \frac{T_{\varepsilon}\left(u_{n}\right)}{\varepsilon} \mid \xi \\
& -\widetilde{\gamma}_{L} \int_{Q_{T}^{n} \cap\left\{\left|u_{n}\right| \leq L\right\}}\left|\nabla T_{L}\left(u_{n}\right)\right|^{p} \xi-\int_{Q_{T}^{n} \cap\left\{\left|u_{n}\right| \leq L\right\}}\left|\widetilde{g}_{L}(t, x)\right| \xi,
\end{aligned}
$$

As we have already noticed, we can choose $\xi$ such that $\xi=\sigma(\eta)$ and so both $\eta$ and $\xi$ satisfy (4.1). Thus, by using Proposition 3.1 with $\delta=\frac{1}{4 \beta}$, we have

$$
\begin{aligned}
& \quad\left|\int_{Q_{n}^{T}} a\left(t, x, u_{n}, \nabla u_{n}\right) \cdot \nabla \xi \frac{T_{\varepsilon}}{\varepsilon}\left(u_{n}\right)\right| \\
& \leq \frac{1}{4} \int_{Q_{n}^{T}} h\left(\left|\nabla u_{n}\right|^{p-1}\right) \xi+C \operatorname{meas}\left(B_{R+\rho}\right) .
\end{aligned}
$$

Hence, dropping positive terms, we have

$$
\begin{gathered}
\frac{1}{4} \int_{Q_{n}^{T}} h\left(\left|\nabla u_{n}\right|^{p-1}\right) \xi+\frac{1}{2} \int_{Q_{n}^{T}}\left|g\left(t, x, u_{n}, \nabla u_{n}\right)\right|\left|\frac{T_{\varepsilon}\left(u_{n}\right)}{\varepsilon}\right| \xi \\
\leq \widetilde{\gamma}_{L} \int_{B_{n} \cap\left\{\left|u_{n}\right| \leq L\right\}}\left|\nabla T_{L}\left(u_{n}\right)\right|^{p} \xi+\int_{B_{n} \cap\left\{\left|u_{n}\right| \leq L\right\}}\left|g_{L}(t, x)\right| \xi \\
+\int_{B_{n}}\left|u_{n}^{0}(x)\right| \xi+C \operatorname{meas}\left(B_{R+\rho}\right),
\end{gathered}
$$

and, by (4.8) and (2.6), the right hand side of the previous inequality is uniformly bounded (with respect to $n$ ). Thus, letting $\varepsilon \rightarrow 0$, Fatou Lemma yields

$$
\int_{Q_{R}^{T}} h\left(\left|\nabla u_{n}\right|^{p-1}\right)+\int_{Q_{R}^{T}}\left|g\left(t, x, u_{n}, \nabla u_{n}\right)\right| \leq C_{R} .
$$

Equiintegrability of the lower order term and uniform estimates on stripes. Let us choose $\psi=\gamma_{j}\left(u_{n}\right) \xi, \forall j>L$, in (4.3) where $\gamma_{j}(s)=T_{1}\left(G_{j}(s)\right)$, and moreover we denote by $\Gamma_{j}(s)=\int_{0}^{s} \gamma_{j}(t) d t$; we note that

$$
\left|G_{j+1}(s)\right| \leq \Gamma_{j}(s) \leq\left|G_{j}(s)\right| .
$$

Thus we have:

$$
\begin{gathered}
\int_{0}^{T}\left\langle\left(u_{n}\right)_{t}, \gamma_{j}\left(u_{n}\right) \xi\right\rangle+\int_{Q_{n}^{t}} a\left(t, x, u_{n}, \nabla u_{n}\right) \cdot \nabla \xi \gamma_{j}\left(u_{n}\right) \\
\quad+\int_{Q_{n}^{t}} a\left(t, x, u_{n}, \nabla u_{n}\right) \cdot \nabla u_{n} \gamma_{j}^{\prime}\left(u_{n}\right) \xi \\
+\int_{Q_{n}^{t}} g\left(t, x, u_{n}, \nabla u_{n}\right) \gamma_{j}\left(u_{n}\right) \xi=\int_{Q_{n}^{t}} f_{n}(t, x) \gamma_{j}\left(u_{n}\right) \xi .
\end{gathered}
$$

Thus, since $j>L$, using that $|\gamma(s)| \leq 1$ and (2.2) we get

$$
\begin{gathered}
\int_{B_{n}} \Gamma_{j}\left(\left|u_{n}(t, x)\right|\right) \xi+\int_{Q_{n}^{t}} a\left(t, x, u_{n}, \nabla u_{n}\right) \cdot \nabla \xi \gamma_{j}\left(u_{n}\right) \\
+\int_{Q_{n}^{t} \cap\left\{j \leq\left|u_{n}\right| \leq j+1\right\}} a\left(t, x, u_{n}, \nabla u_{n}\right) \cdot \nabla u_{n} \xi \\
+\frac{1}{2} \int_{Q_{n}^{t} \cap\left\{\left|u_{n}\right| \geq j\right\}} h\left(\left|\nabla u_{n}\right|^{p-1}\right)\left|\gamma_{j}\left(u_{n}\right)\right| \xi+\frac{1}{2} \int_{Q_{n}^{t} \cap\left\{\left|u_{n}\right| \geq j\right\}}\left|g\left(t, x, u_{n}, \nabla u_{n}\right)\right|\left|\gamma_{j}\left(u_{n}\right)\right| \xi \\
\leq \int_{Q_{n}^{t} \cap\left\{\left|u_{n}\right| \geq j\right\}}\left|f_{n}(t, x)\right| \xi+\int_{B_{n}} \Gamma_{j}\left(\left|u_{n}^{0}(x)\right|\right) \xi .
\end{gathered}
$$


On the other hand by (2.3), and choosing $\xi=\sigma(\eta)$ as above, we deduce by Proposition 3.1 applied with $\delta=\frac{1}{2 \beta}$,

$$
\begin{aligned}
& \int_{Q_{n}^{t} \cap\left\{\left|u_{n}\right| \geq j\right\}}\left|a\left(t, x, u_{n}, \nabla u_{n}\right) \cdot \nabla \xi\right|\left|\gamma_{j}\left(u_{n}\right)\right| \\
& \leq \frac{1}{2} \int_{Q_{n}^{t} \cap\left\{\left|u_{n}\right| \geq j\right\}} h\left(\left|\nabla u_{n}\right|^{p-1}\right)\left|\gamma_{j}\left(u_{n}\right)\right| \xi \\
& +C \operatorname{meas}\left\{(t, x) \in(0, T) \times B_{R+\rho}:\left|u_{n}\right| \geq j\right\},
\end{aligned}
$$

and the last term tends to 0 (uniformly with respect to $n$ ) as $j$ diverges by (4.9). Moreover, by using (4.11) we deduce, dropping the positive term,

$$
\begin{gathered}
\int_{B_{n}} G_{j+1}\left(\left|u_{n}(t, x)\right|\right) \xi+\int_{Q_{n}^{t} \cap\left\{j \leq\left|u_{n}\right| \leq j+1\right\}} a\left(t, x, u_{n}, \nabla u_{n}\right) \cdot \nabla u_{n} \xi \\
\quad+\frac{1}{2} \int_{Q_{n}^{t} \cap\left\{\left|u_{n}\right| \geq j\right\}}\left|g\left(t, x, u_{n}, \nabla u_{n}\right)\right|\left|\gamma_{j}\left(u_{n}\right)\right| \xi \\
\leq \int_{Q_{n}^{t} \cap\left\{\left|u_{n}\right| \geq j\right\}}\left|f_{n}(t, x)\right| \xi+\int_{B_{n}} G_{j}\left(\left|u_{n}^{0}(x)\right|\right) \xi+\varepsilon(j)
\end{gathered}
$$

Since both $u_{0}^{n}(x) \xi$ and $f_{n}(t, x) \xi$ are strongly compact in $L^{1}\left(B_{R+\rho}\right)$ and $L^{1}\left(Q_{R+\rho}^{T}\right)$ respectively, we obtain, dropping positive terms,

$$
\begin{aligned}
\liminf _{j \rightarrow \infty} \sup _{n \in \mathbb{N}} & {\left[\int_{Q_{n}^{T} \cap\left\{j \leq\left|u_{n}\right| \leq j+1\right\}} a\left(t, x, u_{n}, \nabla u_{n}\right) \cdot \nabla u_{n} \xi\right.} \\
+ & \left.\int_{Q_{n}^{T} \cap\left\{\left|u_{n}\right| \geq j+1\right\}}\left|g\left(t, x, u_{n}, \nabla u_{n}\right)\right| \xi\right]=0 .
\end{aligned}
$$

Note that the above estimate, in fact, allows us to say, using (2.7) and since $h(s)$ is superlinear at infinity, that

$$
\sup _{n \in \mathbb{N}} \int_{Q_{R}^{T} \cap\left\{\left|u_{n}\right| \geq j\right\}}\left|\nabla u_{n}\right|^{p-1}=\varepsilon(j) .
$$

Strong convergence of truncations. Let $\varphi_{\lambda}(s)$ be the function introduced in (2.20), where $\lambda>0$ will be fixed in the sequel. We set $T_{k}(u)_{\nu}=\eta_{\nu}\left(T_{k}(u), T_{k}\left(u_{0}\right)\right)$, where $\eta_{\nu}(\cdot)$ has been defined in Lemma 3.3 .

Let us choose $\psi=\varphi_{\lambda}\left(z_{n, \nu}\right) S_{j}^{\prime}\left(u_{n}\right) \xi$ as test function in (4.3), where $z_{n, \nu}=$ $T_{k}\left(u_{n}\right)-T_{k}(u)_{\nu}, k \geq L$, and $S_{j}(s)$ is as in (2.21). Thus we have

$$
\begin{gathered}
\int_{0}^{T}\left\langle S_{j}\left(u_{n}\right)_{t}, \varphi_{\lambda}\left(z_{n, \nu}\right) \xi\right\rangle+\int_{Q_{n}^{T}} a\left(t, x, u_{n}, \nabla u_{n}\right) \cdot \nabla \xi \varphi_{\lambda}\left(z_{n, \nu}\right) S_{j}^{\prime}\left(u_{n}\right) \\
+\int_{Q_{n}^{T}} a\left(t, x, u_{n}, \nabla u_{n}\right) \cdot \nabla\left(T_{k}\left(u_{n}\right)-T_{k}(u)_{\nu}\right) \varphi_{\lambda}^{\prime}\left(z_{n, \nu}\right) S_{j}^{\prime}\left(u_{n}\right) \xi \\
\quad+\int_{Q_{n}^{T}} a\left(t, x, u_{n}, \nabla u_{n}\right) \cdot \nabla u_{n} \varphi_{\lambda}\left(z_{n, \nu}\right) S_{j}^{\prime \prime}\left(u_{n}\right) \xi \\
+\int_{Q_{n}^{T}} g\left(t, x, u_{n}, \nabla u_{n}\right) \varphi_{\lambda}\left(z_{n, \nu}\right) S_{j}^{\prime}\left(u_{n}\right) \xi=\int_{Q_{n}^{T}} f_{n}(t, x) \varphi_{\lambda}\left(z_{n, \nu}\right) S_{j}^{\prime}\left(u_{n}\right) \xi .
\end{gathered}
$$

We first note that

$$
\int_{Q_{n}^{T}} a\left(t, x, u_{n}, \nabla u_{n}\right) \cdot \nabla\left(T_{k}\left(u_{n}\right)-T_{k}(u)_{\nu}\right) \varphi_{\lambda}^{\prime}\left(z_{n, \nu}\right) S_{j}^{\prime}\left(u_{n}\right) \xi
$$




$$
\begin{gathered}
=\int_{Q_{n}^{T} \cap\left\{\left|u_{n}\right| \leq k\right\}} a\left(t, x, u_{n}, \nabla u_{n}\right) \cdot \nabla\left(T_{k}\left(u_{n}\right)-T_{k}(u)_{\nu}\right) \varphi_{\lambda}^{\prime}\left(z_{n, \nu}\right) S_{j}^{\prime}\left(u_{n}\right) \xi \\
-\int_{Q_{n}^{T} \cap\left\{\left|u_{n}\right| \geq k\right\}} a\left(t, x, u_{n}, \nabla u_{n}\right) \cdot \nabla T_{k}(u)_{\nu} \varphi_{\lambda}^{\prime}\left(z_{n, \nu}\right) S_{j}^{\prime}\left(u_{n}\right) \xi .
\end{gathered}
$$

Using (4.8) and recalling that $\operatorname{Supp}\left(S_{j}^{\prime}\right) \subset[-j-1, j+1]$, there exists $\varsigma_{k, j} \in$ $\left(L^{p^{\prime}}\left(Q_{R}^{T}\right)\right)^{N+1}$ such that,

$$
\begin{gathered}
\lim _{n \rightarrow \infty} \int_{Q_{n}^{T} \cap\left\{\left|u_{n}\right| \geq k\right\}} a\left(t, x, u_{n}, \nabla u_{n}\right) \cdot \nabla T_{k}(u)_{\nu} \varphi_{\lambda}^{\prime}\left(z_{n, \nu}\right) S_{j}^{\prime}\left(u_{n}\right) \xi \\
=\int_{Q_{R+\rho}^{T} \cap\{|u| \geq k\}} \varsigma_{k, j} \cdot \nabla T_{k}(u)_{\nu} \varphi_{\lambda}^{\prime}\left(z_{\nu}\right) \xi,
\end{gathered}
$$

and last integral tends to 0 as $\nu$ diverges. In fact we have that $T_{k}(u)_{\nu} \rightarrow T_{k}(u)$ strongly in $L^{p}\left(0, T ; W_{\text {loc }}^{1, p}\left(\mathbb{R}^{N}\right)\right)$, and consequently $\left|\nabla T_{k}(u)_{\nu}\right| \chi_{\{|u| \geq k\}}$ tends to zero strongly in $L^{p}\left(0, T ; L_{\text {loc }}^{p}\left(\mathbb{R}^{N}\right)\right)$. Thus

$$
\begin{gathered}
\int_{Q_{n}^{T}} a\left(t, x, u_{n}, \nabla u_{n}\right) \cdot \nabla\left(T_{k}\left(u_{n}\right)-T_{k}(u)_{\nu}\right) \varphi_{\lambda}^{\prime}\left(z_{n, \nu}\right) S_{j}^{\prime}\left(u_{n}\right) \xi \\
=\int_{Q_{n}^{T}} a\left(t, x, u_{n}, \nabla T_{k}\left(u_{n}\right)\right) \cdot \nabla\left(T_{k}\left(u_{n}\right)-T_{k}(u)_{\nu}\right) \varphi_{\lambda}^{\prime}\left(z_{n, \nu}\right) S_{j}^{\prime}\left(u_{n}\right) \xi+\varepsilon(n, \nu) .
\end{gathered}
$$

On the other hand, since $k \geq L$,

$$
\begin{gathered}
\int_{Q_{n}^{T}} g\left(t, x, u_{n}, \nabla u_{n}\right) \varphi_{\lambda}\left(z_{n, \nu}\right) S_{j}^{\prime}\left(u_{n}\right) \xi \\
\geq \int_{Q_{n}^{T}} h\left(\left|\nabla u_{n}\right|^{p-1}\right)\left|\varphi_{\lambda}\left(z_{n, \nu}\right)\right| S_{j}^{\prime}\left(u_{n}\right) \xi-\int_{Q_{n}^{T} \cap\left\{\left|u_{n}\right| \leq k\right\}}\left|\widetilde{g}_{k}(t, x)\right|\left|\varphi_{\lambda}\left(z_{n, \nu}\right)\right| \xi \\
-\frac{\widetilde{\gamma}_{k}}{\alpha} \int_{Q_{n}^{T} \cap\left\{\left|u_{n}\right| \leq k\right\}} a\left(t, x, u_{n}, \nabla T_{k}\left(u_{n}\right)\right) \cdot \nabla\left(T_{k}\left(u_{n}\right)-T_{k}(u)_{\nu}\right) S_{j}^{\prime}\left(u_{n}\right)\left|\varphi_{\lambda}\left(z_{n, \nu}\right)\right| \xi \\
+\frac{\gamma_{k}}{\alpha} \int_{Q_{n}^{T}} a\left(t, x, u_{n}, \nabla T_{k}\left(u_{n}\right)\right) \cdot \nabla T_{k}(u)_{\nu} S_{j}^{\prime}\left(u_{n}\right) \varphi_{\lambda}\left(z_{n, \nu}\right) \xi,
\end{gathered}
$$

where, as before, $\widetilde{\gamma}_{k}=\gamma_{k}+c_{1}$ and $\widetilde{g}_{k}=g_{k}+c_{1}$. Reasoning as before,

$$
\frac{\gamma_{k}}{\alpha} \int_{Q_{n}^{T}} a\left(t, x, u_{n}, \nabla T_{k}\left(u_{n}\right)\right) \cdot \nabla T_{k}(u)_{\nu} \varphi_{\lambda}\left(z_{n, \nu}\right) S_{j}^{\prime}\left(u_{n}\right) \xi=\varepsilon(n, \nu),
$$

and

$$
\int_{Q_{n}^{T}}\left[\left|f_{n}(t, x)\right|+\left|\widetilde{g}_{k}(t, x)\right|\right] \varphi_{\lambda}\left(z_{n, \nu}\right) S_{j}^{\prime}\left(u_{n}\right) \xi=\varepsilon(n, \nu)
$$


Gathering the above informations together, we deduce:

$$
\begin{gathered}
\int_{0}^{T}\left\langle S_{j}\left(u_{n}\right)_{t}, \varphi_{\lambda}\left(z_{n, \nu}\right) \xi\right\rangle+\int_{Q_{n}^{T}} a\left(t, x, u_{n}, \nabla u_{n}\right) \cdot \nabla \xi \varphi_{\lambda}\left(z_{n, \nu}\right) S_{j}^{\prime}\left(u_{n}\right) \\
+\int_{Q_{n}^{T}} a\left(t, x, u_{n}, \nabla T_{k}\left(u_{n}\right)\right) \cdot \nabla\left(T_{k}\left(u_{n}\right)-T_{k}(u)_{\nu}\right) \varphi_{\lambda}^{\prime}\left(z_{n, \nu}\right) S_{j}^{\prime}\left(u_{n}\right) \xi \\
\quad+\int_{Q_{n}^{T}} a\left(t, x, u_{n}, \nabla u_{n}\right) \cdot \nabla u_{n} \varphi_{\lambda}\left(z_{n, \nu}\right) S_{j}^{\prime \prime}\left(u_{n}\right) \xi \\
-\frac{\widetilde{\gamma}_{k}}{\alpha} \int_{Q_{n}^{T}} a\left(t, x, u_{n}, \nabla T_{k}\left(u_{n}\right)\right) \cdot \nabla\left(T_{k}\left(u_{n}\right)-T_{k}(u)_{\nu}\right) \varphi_{\lambda}\left(z_{n, \nu}\right) S_{j}^{\prime}\left(u_{n}\right) \xi \\
\quad+\int_{Q_{n}^{T}} h\left(\left|\nabla u_{n}\right|^{p-1}\right)\left|\varphi_{\lambda}\left(z_{n, \nu}\right)\right| S_{j}^{\prime}\left(u_{n}\right) \xi \leq \varepsilon(n, \nu) .
\end{gathered}
$$

Moreover, using that $\xi=\sigma(\eta), \eta$ chosen as in (4.1) and thanks to Proposition 3.1. the second integral in (4.15) is estimated as

$$
\left|\int_{Q_{n}^{T}} a\left(t, x, u_{n}, \nabla u_{n}\right) \cdot \nabla \xi \varphi_{\lambda}\left(z_{n, \nu}\right)\right| \leq \frac{1}{2} \int_{Q_{n}^{T}} h\left(\left|\nabla u_{n}\right|^{p-1}\right)\left|\varphi_{\lambda}\left(z_{n, \nu}\right)\right| S_{j}^{\prime}\left(u_{n}\right) \xi+\varepsilon(n, \nu) .
$$

Thus by (4.15), dropping positive terms, we have

$$
\begin{gathered}
\int_{0}^{T}\left\langle S_{j}\left(u_{n}\right)_{t}, \varphi_{\lambda}\left(z_{n, \nu}\right) \xi\right\rangle \\
+\int_{Q_{n}^{T}} a\left(t, x, u_{n}, \nabla T_{k}\left(u_{n}\right)\right) \cdot \nabla z_{n, \nu}\left[\varphi_{\lambda}^{\prime}\left(z_{n, \nu}\right)-\frac{\widetilde{\gamma}_{k}}{\alpha} \varphi_{\lambda}\left(z_{n, \nu}\right)\right] S_{j}^{\prime}\left(u_{n}\right) \xi \\
+\int_{Q_{n}^{T}} a\left(t, x, u_{n}, \nabla u_{n}\right) \cdot \nabla u_{n} \varphi_{\lambda}\left(z_{n, \nu}\right) S_{j}^{\prime \prime}\left(u_{n}\right) \xi \leq \varepsilon(n, \nu) .
\end{gathered}
$$

Noticing that, by definition of $T_{k}(u)_{\nu}$,

$$
-\int_{Q_{n}^{T}} a\left(t, x, u_{n}, \nabla T_{k}(u)_{\nu}\right) \cdot \nabla z_{n, \nu}\left[\varphi_{\lambda}^{\prime}\left(z_{n, \nu}\right)-\frac{\widetilde{\gamma}_{k}}{\alpha}\left|\varphi_{\lambda}\left(z_{n, \nu}\right)\right|\right] S_{j}^{\prime}\left(u_{n}\right) \xi=\varepsilon(n, \nu),
$$

we can add this quantity in both sides of (4.16). Moreover, by (4.12), we get

$$
\left|\int_{Q_{n}^{T}} a\left(t, x, u_{n}, \nabla u_{n}\right) \cdot \nabla u_{n} \varphi_{\lambda}\left(z_{n, \nu}\right) S_{j}^{\prime \prime}\left(u_{n}\right) \xi\right| \leq \varepsilon(j) .
$$

Finally, in order to get rid of the integral involving the time derivative of $S_{j}\left(u_{n}\right)$, we apply the following inequality, whose proof is postponed at the end of this Section.

Claim. $\forall j \geq j_{0}$ :

$$
\int_{0}^{t}\left\langle S_{j}\left(u_{n}\right)_{t}, \varphi_{\lambda}\left(z_{n, \nu}\right) \xi\right\rangle \geq \varepsilon(n, \nu)
$$

Using (4.17) in (4.16) we deduce that, for $j$ large enough,

$$
\begin{aligned}
& \int_{Q_{n}^{T}}\left(a\left(t, x, u_{n}, \nabla T_{k}\left(u_{n}\right)\right)-a\left(t, x, u_{n}, \nabla T_{k}(u)_{\nu}\right)\right) \cdot \nabla z_{n, \nu} \\
& \quad \times\left[\varphi_{\lambda}^{\prime}\left(z_{n, \nu}\right)-\frac{\widetilde{\gamma}_{k}}{\alpha} \varphi_{\lambda}^{\prime}\left(z_{n, \nu}\right)\right] S_{j}^{\prime}\left(u_{n}\right) \xi \leq \varepsilon(n, \nu)+\varepsilon(j) .
\end{aligned}
$$


By a suitable choice of $\lambda$ (according with (2.20) applied with $a=1$ and $b=\frac{\widetilde{\gamma} k}{\alpha}$ ) we deduce that

$$
\int_{Q_{n}^{T}}\left(a\left(t, x, u_{n}, \nabla T_{k}\left(u_{n}\right)\right)-a\left(t, x, u_{n}, \nabla T_{k}(u)_{\nu}\right)\right) \cdot \nabla z_{n, \nu} S_{j}^{\prime}\left(u_{n}\right) \xi \leq \varepsilon(n, \nu)+\varepsilon(j) .
$$

Lemma 5 in 9 yields

$$
T_{k}\left(u_{n}\right) \rightarrow T_{k}(u) \text { strongly in } L^{p}\left(0, T ; W^{1, p}\left(B_{R}\right)\right) .
$$

Note that the above convergence implies that, up to subsequences, $\nabla T_{k}\left(u_{n}\right)$ a.e. converges to $\nabla T_{k}(u)$, and, by a diagonal argument, we conclude that (again up to not relabeled subsequences)

$$
\nabla u_{n} \rightarrow \nabla u \quad \text { a.e. . }
$$

Moreover combining (4.18) with (4.13) and (4.19) we deduce, using Vitali Theorem, that

$$
\left|\nabla u_{n}\right|^{p-1} \rightarrow|\nabla u|^{p-1} \quad \text { strongly in } L^{1}\left(0, T ; L_{\text {loc }}^{1}\left(\mathbb{R}^{N}\right)\right),
$$

and by (4.12), (4.18) and (4.19) we have

$$
g\left(x, u_{n}, \nabla u_{n}\right) \xi \rightarrow g(x, u, \nabla u) \xi \text { strongly in } L^{1}\left((0, T) \times \mathbb{R}^{N}\right) .
$$

Passing to the limit. Let us choose $\psi=\phi(t, x) S^{\prime}\left(u_{n}\right)$ in (4.3) where $\phi$ is in $C_{0}^{1}\left([0, T) \times \mathbb{R}^{N}\right)$ and $S$ is as in Definition 2.2. We have:

$$
\begin{gathered}
\int_{0}^{T}\left\langle\left(u_{n}\right)_{t}, \phi S^{\prime}\left(u_{n}\right)\right\rangle+\int_{Q_{n}^{T}} a\left(t, x, u_{n}, \nabla u_{n}\right) \cdot \nabla \phi S^{\prime}\left(u_{n}\right) \\
+\int_{Q_{n}^{T}} a\left(t, x, u_{n}, \nabla u_{n}\right) \cdot \nabla u_{n} S^{\prime \prime}\left(u_{n}\right) \phi \\
+\int_{Q_{n}^{T}} g\left(t, x, u_{n}, \nabla u_{n}\right) \phi=\int_{Q_{n}^{T}} f_{n}(t, x) \phi .
\end{gathered}
$$

We first note that there exists $R>0$ such that $\operatorname{supp} \phi(x, t) \subset(0, T) \times B_{R}$, so that, integrating by parts, and recalling that $\phi(T, x)=0$ we get:

$$
\begin{gathered}
\lim _{n \rightarrow+\infty} \int_{0}^{T}\left\langle\left(u_{n}\right)_{t}, \phi S^{\prime}\left(u_{n}\right)\right\rangle \\
=\lim _{n \rightarrow+\infty} \int_{\mathbb{R}^{N}} S\left(u_{n}\right) \phi(T, x)-\int_{\mathbb{R}^{N}} S\left(u_{n}^{0}\right) \phi(0, x)-\int_{Q^{T}} S\left(u_{n}\right) \phi_{t}(t, x) \\
=-\int_{\mathbb{R}^{N}} S\left(u_{0}\right) \phi(0, x)-\int_{Q^{T}} S(u) \phi_{t}(t, x),
\end{gathered}
$$

where $Q^{T}=(0, T) \times \mathbb{R}^{N}$. Moreover, by (2.3) and (4.20), we have

$$
\lim _{n \rightarrow+\infty} \int_{Q_{n}^{T}} a\left(t, x, u_{n}, \nabla u_{n}\right) \cdot \nabla \phi S^{\prime}\left(u_{n}\right)=\int_{Q_{n}^{T}} a(t, x, u, \nabla u) \cdot \nabla \phi S^{\prime}(u),
$$

while by (4.18) and (4.21), (4.12) we deduce that both

$$
\lim _{n \rightarrow+\infty} \int_{Q_{n}^{T}} a\left(t, x, u_{n}, \nabla u_{n}\right) \cdot \nabla u_{n} S^{\prime \prime}\left(u_{n}\right) \phi=\int_{Q_{n}^{T}} a(t, x, u, \nabla u) \cdot \nabla u S^{\prime \prime}(u) \phi,
$$


and

$$
\lim _{n \rightarrow+\infty} \int_{Q_{n}^{T}} g\left(t, x, u_{n}, \nabla u_{n}\right) S^{\prime}\left(u_{n}\right) \phi=\int_{Q_{n}^{T}} g(t, x, u, \nabla u) S^{\prime}(u) \phi .
$$

Finally, since $f_{n} \xi \rightarrow f \xi$ in $L^{1}\left(Q^{T}\right)$ we can pass to the limit in the last integral in (4.22). Consequently $u(t, x)$ is a solution for (2.12) in the sense of Definition 2.2.

Continuity with values in $L_{l o c}^{1}$. We prove that $u_{n}$ converges up to subsequences (not relabeled) toward $u$ in $C^{0}\left([0, T] ; L_{l o c}^{1}\left(\mathbb{R}^{N}\right)\right)$ by using a classical monotonicity argument.

For any integers $n$ and $m$, let us consider the formulations of (4.3) with indexes $n$ and $m$, respectively. For any $R, \rho>0$, let us multiply the formulation of both $u_{n}$ and $u_{m}$ by $T_{1}\left(u_{n}-u_{m}\right) \xi$, with $n, m>R+\rho$, where $\xi$ has been defined in (4.1). By subtracting the two resulting identities, and dropping the energy terms (by (2.4)), we get:

$$
\begin{gathered}
\sup _{t \in[0, T]} \int_{B_{R}} \Theta_{1}\left(u_{n}-u_{m}\right)(t, x) \\
\leq\left\|a\left(t, x, u_{n}, \nabla u_{n}\right)-a\left(t, x, u_{m}, \nabla u_{n}\right) \cdot \nabla \xi\right\|_{L^{1}\left(Q_{R+\rho}^{T}\right)}+\left\|u_{n}^{0}-u_{m}^{0}\right\|_{L^{1}\left(B_{R+\rho}\right)} \\
+\left\|g\left(t, x, u_{n}, \nabla u_{n}\right)-g\left(t, x, u_{m}, \nabla u_{m}\right)\right\|_{L^{1}\left(Q_{R+\rho}^{T}\right)}+\left\|f_{n}-f_{m}\right\|_{L^{1}\left(Q_{R+\rho}^{T}\right)} .
\end{gathered}
$$

Note that by (4.20) and (4.21) and using that both $\left\{u_{n}^{0}\right\}_{n \in \mathbb{N}}$ and $\left\{f_{n}\right\}_{n \in \mathbb{N}}$ are strongly compact in $L^{1}\left(Q_{R+\rho}^{T}\right)$ and $L^{1}\left(B_{R+\rho}\right)$, respectively, the right hand side above converges to 0 as $n$ and $m$ diverge. Hence it follows that, up to subsequences (not relabeled), $u_{n}$ is a Cauchy sequence in $C^{0}\left([0, T] ; L^{1}\left(B_{R}\right)\right)$.

To complete the proof of Theorem 2.3 we need to prove that inequality (4.17) holds: the proof follows the outlines of Lemma 3.2 in [13].

of 4.17$)$. We recall that, by previous estimates, $T_{k}\left(u_{n}\right)$ converges to $T_{k}(u)$ weakly in $L^{p}\left(0, T ; W_{\text {loc }}^{1, p}\left(\mathbb{R}^{N}\right)\right)$. Here we exploit an approximation argument by using Lemma 3.3. We set, for every $\sigma>0, u_{n, \sigma}=\eta_{\sigma}\left(u_{n}, u_{n}^{0}\right)$; we know that $u_{n, \sigma} \in L^{p}\left(0, T ; W_{0}^{1, p}\left(B_{n}\right)\right),\left(u_{n, \sigma}\right)_{t} \in L^{p}\left(0, T ; W_{0}^{1, p}\left(B_{n}\right)\right)$, and moreover, both

$$
u_{n, \sigma} \longrightarrow u_{n} \text { in } L^{p}\left(0, T ; W_{0}^{1, p}\left(B_{n}\right)\right),
$$

and

$$
\left(u_{n, \sigma}\right)_{t} \longrightarrow\left(u_{n}\right)_{t} \text { in } L^{p^{\prime}}\left(0, T ; W^{-1, p^{\prime}}\left(B_{n}\right)\right)+L^{1}\left(Q_{n}^{T}\right),
$$

with $u_{n, \sigma}(0, x)=u_{n}^{0}$.

This approximation argument will allow us to consider derivatives with respect to $t$ of the composition between Lipschitz functions and $u_{n, \sigma}$. Thanks to these properties we have that

$$
\begin{gathered}
\int_{0}^{T}\left\langle S_{j}\left(u_{n}\right)_{t}, \varphi_{\lambda}\left(z_{n, \nu}\right) \xi\right\rangle \\
=\lim _{\sigma \rightarrow 0} \int_{0}^{T}\left\langle S_{j}\left(u_{n, \sigma}\right)_{t}, \varphi_{\lambda}\left(T_{k}\left(u_{n, \sigma}\right)-T_{k}(u)_{\nu}\right) \xi\right\rangle
\end{gathered}
$$

Our aim is to prove that

$$
\int_{0}^{T}\left\langle S_{j}\left(u_{n, \sigma}\right)_{t}, \varphi_{\lambda}\left(T_{k}\left(u_{n, \sigma}\right)-T_{k}(u)_{\nu}\right) \xi\right\rangle \geq \varepsilon(n, \nu) .
$$


Note that, for any $j>k$, we can write

$$
S_{j}\left(u_{n, \sigma}\right)=T_{k}\left(u_{n, \sigma}\right)+G_{k}\left(S_{j}\left(u_{n, \sigma}\right)\right)
$$

thus, if we define $\phi_{\lambda}(s)=\int_{0}^{s} \varphi_{\lambda}$, we have

$$
\begin{gathered}
\int_{0}^{T}\left\langle S_{j}\left(u_{n, \sigma}\right)_{t}, \varphi_{\lambda}\left(T_{k}\left(u_{n, \sigma}\right)-T_{k}(u)_{\nu}\right) \xi\right\rangle \\
=\int_{B_{R}} \phi_{\lambda}\left(T_{k}\left(u_{n, \sigma}\right)-T_{k}(u)_{\nu}\right)(T) \xi-\int_{B_{R}} \phi_{\lambda}\left(T_{k}\left(u_{n, \sigma}\right)-T_{k}(u)_{\nu}\right)(0) \xi \\
+\int_{0}^{T}\left\langle G_{k}\left(S_{j}\left(u_{n, \sigma}\right)\right)_{t}, \varphi_{\lambda}\left(T_{k}\left(u_{n, \sigma}\right)-T_{k}(u)_{\nu}\right) \xi\right\rangle \\
+\int_{Q_{R}^{T}} \nu\left(T_{k}(u)-T_{k}(u)_{\nu}\right) \varphi_{\lambda}\left(T_{k}\left(u_{n, \sigma}\right)-T_{k}(u)_{\nu}\right) \xi
\end{gathered}
$$

where we used that $\left(T_{k}(u)_{\nu}\right)_{t}=\nu\left(T_{k}(u)-T_{k}(u)_{\nu}\right)$. Since both $\phi_{\lambda}(s)>0, \forall s \in \mathbb{R}$ and $T_{k}\left(u_{n}^{0}\right) \xi \rightarrow T_{k}\left(u_{0}\right) \xi$ *-weakly in $L^{\infty}\left(B_{R}\right)$, we deduce that

$$
\int_{B_{R}} \phi_{\lambda}\left(T_{k}\left(u_{n, \sigma}\right)-T_{k}(u)_{\nu}\right)(T) \xi-\int_{B_{R}} \phi_{\lambda}\left(T_{k}\left(u_{n, \sigma}\right)-T_{k}(u)_{\nu}\right)(0) \xi \geq \varepsilon(n, \nu) .
$$

Moreover

$$
\begin{gathered}
\int_{Q_{R}^{T}} \nu\left(T_{k}(u)-T_{k}(u)_{\nu}\right) \varphi_{\lambda}\left(T_{k}\left(u_{n, \sigma}\right)-T_{k}(u)_{\nu}\right) \xi \\
=\int_{Q_{R}^{T}} \nu\left(T_{k}(u)-T_{k}(u)_{\nu}\right) \varphi_{\lambda}\left(T_{k}(u)-T_{k}(u)_{\nu}\right) \xi+\varepsilon(\sigma, n) \geq \varepsilon(\sigma, n),
\end{gathered}
$$

since $s \cdot s e^{\lambda s^{2}} \geq 0$. Finally, we deal with the term

$$
\int_{0}^{T}\left\langle G_{k}\left(S_{j}\left(u_{n, \sigma}\right)\right)_{t}, \varphi_{\lambda}\left(T_{k}\left(u_{n, \sigma}\right)-T_{k}(u)_{\nu}\right) \xi\right\rangle .
$$

Integrating by parts we deduce that

$$
\begin{gathered}
\int_{0}^{T}\left\langle G_{k}\left(S_{j}\left(u_{n, \sigma}\right)\right)_{t}, \varphi_{\lambda}\left(T_{k}\left(u_{n, \sigma}\right)-T_{k}(u)_{\nu}\right) \xi\right\rangle \\
=\int_{B_{R}} G_{k}\left(S_{j}\left(u_{n, \sigma}\right)\right)(T) \varphi_{\lambda}\left(T_{k}\left(u_{n, \sigma}\right)-T_{k}(u)_{\nu}\right)(T) \xi \\
-\int_{B_{R}} G_{k}\left(S_{j}\left(u_{n, \sigma}\right)\right)(0) \varphi_{\lambda}\left(T_{k}\left(u_{n, \sigma}\right)-T_{k}(u)_{\nu}\right)(0) \xi \\
-\int_{0}^{T}\left\langle G_{k}\left(S_{j}\left(u_{n, \sigma}\right)\right) \varphi_{\lambda}^{\prime}\left(T_{k}\left(u_{n, \sigma}\right)-T_{k}(u)_{\nu}\right) \xi,\left(T_{k}\left(u_{n, \sigma}\right)-T_{k}(u)_{\nu}\right)_{t}\right\rangle .
\end{gathered}
$$

Thus the first term on the right hand side is positive since

$$
\begin{aligned}
& \int_{B_{R}} G_{k}\left(S_{j}\left(u_{n, \sigma}\right)\right)(T) \varphi_{\lambda}\left(T_{k}\left(u_{n, \sigma}\right)-T_{k}(u)_{\nu}\right)(T) \xi \\
& =\int_{B_{R} \cap\left\{u_{n, \sigma}>k\right\}} G_{k}\left(S_{j}\left(u_{n, \sigma}\right)\right)(T) \varphi_{\lambda}\left(k-T_{k}(u)_{\nu}\right)(T) \xi \\
& +\int_{B_{R} \cap\left\{u_{n, \sigma}<-k\right\}} G_{k}\left(S_{j}\left(u_{n, \sigma}\right)\right)(T) \varphi_{\lambda}\left(-k-T_{k}(u)_{\nu}\right)(T) \xi \geq 0,
\end{aligned}
$$


while the second term vanishes passing to the limit with respect to $\sigma, n$ and then $\nu$. Concerning the last one, we note that since $G_{k}\left(S_{j}\left(u_{n, \sigma}\right)\right)$ is 0 if $\left|u_{n, \sigma}\right| \leq k$, thus

$$
\begin{gathered}
-\int_{0}^{T}\left\langle G_{k}\left(S_{j}\left(u_{n, \sigma}\right)\right) \varphi_{\lambda}^{\prime}\left(T_{k}\left(u_{n, \sigma}\right)-T_{k}(u)_{\nu}\right) \xi,\left(T_{k}\left(u_{n, \sigma}\right)-T_{k}(u)_{\nu}\right)_{t}\right\rangle \\
=\nu \int_{Q_{R}^{T}} G_{k}\left(S_{j}\left(u_{n, \sigma}\right)\right) \varphi_{\lambda}^{\prime}\left(T_{k}\left(u_{n, \sigma}\right)-T_{k}(u)_{\nu}\right)\left(T_{k}(u)-T_{k}(u)_{\nu}\right) \xi .
\end{gathered}
$$

Finally, taking the limit respectively in $\sigma$ and $n$, we have

$$
\begin{gathered}
-\int_{0}^{T}\left\langle G_{k}\left(S_{j}\left(u_{n, \sigma}\right)\right) \varphi_{\lambda}^{\prime}\left(T_{k}\left(u_{n, \sigma}\right)-T_{k}(u)_{\nu}\right) \xi,\left(T_{k}\left(u_{n, \sigma}\right)-T_{k}(u)_{\nu}\right)_{t}\right\rangle \\
=\nu \int_{Q_{R}^{T}} G_{k}\left(S_{j}(u)\right) \varphi_{\lambda}^{\prime}\left(T_{k}(u)-T_{k}(u)_{\nu}\right)\left(T_{k}(u)-T_{k}(u)_{\nu}\right) \xi+\varepsilon(\sigma, n) \\
\quad=\nu \int_{Q_{R}^{T} \cap\{u>k\}} G_{k}\left(S_{j}(u)\right) \varphi_{\lambda}^{\prime}\left(k-T_{k}(u)_{\nu}\right)\left(k-T_{k}(u)_{\nu}\right) \xi \\
+\nu \int_{Q_{R}^{T} \cap\{u<-k\}} G_{k}\left(S_{j}(u)\right) \varphi_{\lambda}^{\prime}\left(-k-T_{k}(u)_{\nu}\right)\left(-k-T_{k}(u)_{\nu}\right) \xi+\varepsilon(\sigma, n) \geq \varepsilon(\sigma, n)
\end{gathered}
$$

since $\varphi_{\lambda}^{\prime}(s)>0, \forall s \in \mathbb{R}$ and the claim is proved because of (4.23).

This concludes the proof of Theorem 2.3 .

of Theorem 2.5. . Let $u_{n}$ be a weak solutions of the following problem

$$
\begin{cases}\left(u_{n}\right)_{t}-\operatorname{div} a\left(t, x, u_{n}, \nabla u_{n}\right)+g\left(t, x, u_{n}, \nabla u_{n}\right)=f_{n}(t, x) & \text { in } Q_{\Omega}^{T}, \\ u_{n}(t, x)=n & \text { on } \partial_{\mathcal{P}} Q_{\Omega}^{T}, \\ u_{n}(x, 0)=u_{n}^{0}(x) & \text { in } \Omega,\end{cases}
$$

where $f_{n}(t, x)=T_{n}(f(t, x))$ and $u_{n}^{0}(x)=T_{n}\left(u_{0}(x)\right)$. The existence of a weak solution for (4.2) is still a consequence of the result of [13]. This means that there exists a function $u_{n}$ such that $u_{n}-n \in L^{p}\left(0, T ; W_{0}^{1, p}(\Omega)\right),\left(u_{n}\right)_{t} \in L^{p^{\prime}}\left(0, T ; W^{-1, p^{\prime}}(\Omega)\right)$, $g\left(t, x, u_{n}, \nabla u_{n}\right) \in L^{1}((0, T) \times \Omega)$, and the following identity holds true

$$
\begin{gathered}
\int_{0}^{T}\left\langle\left(u_{n}\right)_{t}, \psi\right\rangle+\int_{Q_{\Omega}^{T}} a\left(t, x, u_{n}, \nabla u_{n}\right) \cdot \nabla \psi \\
\quad+\int_{Q_{\Omega}^{T}} g\left(t, x, u_{n}, \nabla u_{n}\right) \psi=\int_{Q_{\Omega}^{T}} f_{n} \psi, \\
\forall \psi \in L^{p}\left(0, T ; W_{0}^{1, p}(\Omega)\right) \cap L^{\infty}\left(Q_{\Omega}^{T}\right) .
\end{gathered}
$$

The idea of the proof is similar to the one of Theorem 2.3. The main difference relies on the fact that now we need to have an information about $u_{n}$ (and consequently $u$ ) at the boundary, and so we need first to prove a global (i.e. on the whole $\Omega$ ) estimate on the truncates in the energy space. On the other hand, for the second part of the proof, we follow exactly the same outline of the one of Theorem 2.3. Indeed, the estimates proved there are localized in $(0, T) \times B_{R}, \forall R>0$. Since, in order to pass to the limit in the equation, we need to use such estimates on any compact subset $\varpi \subset \subset(0, T) \times \Omega$, we observe that there exists $\omega \subset \subset \Omega$ such that $\varpi \subset \subset(0, T) \times \omega$. Thus

$$
\exists M \in \mathbb{N}, x_{i} \in \Omega, r_{i}>0, i=1, \ldots, M, \text { such that } \omega \subset \bigcup_{i=1}^{M} B_{r_{i}}\left(x_{i}\right) .
$$


It is clear that it is enough to prove all the estimates on a ball and without loss of generality we can suppose that it is centered at the origin.

Global estimate on truncations. Let us choose, $\forall n \geq k \geq L, \psi=\varphi_{\lambda}\left(T_{k}\left(u_{n}\right)-k\right)$ as test function in (4.25), with $\lambda>0$ to be fixed later. Thus we have

$$
\begin{gathered}
\int_{\Omega} \Upsilon_{\lambda, k}\left(u_{n}(t, x)\right)-\int_{\Omega} \Upsilon_{\lambda, k}\left(u_{n}^{0}(x)\right) \\
+\int_{Q_{\Omega}^{T}} a\left(t, x, u_{n}, \nabla u_{n}\right) \cdot \nabla T_{k}\left(u_{n}\right) \varphi_{\lambda}^{\prime}\left(T_{k}\left(u_{n}\right)-k\right) \\
\quad+\int_{Q_{\Omega}^{T}} g\left(t, x, u_{n}, \nabla u_{n}\right) \varphi_{\lambda}\left(T_{k}\left(u_{n}\right)-k\right) \\
=\int_{Q_{\Omega}^{T}} f_{n}^{+} \varphi_{\lambda}\left(T_{k}\left(u_{n}\right)-k\right)-\int_{Q_{\Omega}^{T}} f_{n}^{-} \varphi_{\lambda}\left(T_{k}\left(u_{n}\right)-k\right),
\end{gathered}
$$

where

$$
\Upsilon_{\lambda, k}(s)= \begin{cases}-2 k e^{4 \lambda k^{2}}(s+k)+\frac{1}{2 \lambda}\left[e^{4 \lambda k^{2}}-e^{\lambda k^{2}}\right] & \text { if } s<-k, \\ \frac{1}{2 \lambda}\left[e^{\lambda(s-k)^{2}}-e^{\lambda k^{2}}\right] & \text { if }-k \leq s<k, \\ \frac{1}{2 \lambda}\left[1-e^{\lambda k^{2}}\right] & \text { if } s \geq k,\end{cases}
$$

is a primitive of $\varphi_{\lambda}\left(T_{k}(s)-k\right)$. Note that, since $\Upsilon_{\lambda, k}(s)$ is decreasing and $\Upsilon_{\lambda, k}(0)=$ 0 , then

$$
\begin{gathered}
\int_{\Omega} \Upsilon_{\lambda, k}\left(u_{n}(t, x)\right)-\int_{\Omega} \Upsilon_{\lambda, k}\left(u_{n}^{0}(x)\right) \\
\geq \int_{\Omega \cap\left\{0 \leq u_{n} \leq k\right\}} \Upsilon_{\lambda, k}\left(u_{n}(t, x)\right)+\int_{\Omega \cap\left\{u_{n}>k\right\}} \Upsilon_{\lambda, k}\left(u_{n}(t, x)\right) \\
-\int_{\Omega \cap\left\{u_{n}^{0} \leq-k\right\}} \Upsilon_{\lambda, k}\left(u_{n}^{0}(x)\right)-\int_{\Omega \cap\left\{-k \leq u_{n}^{0} \leq 0\right\}} \Upsilon_{\lambda, k}\left(u_{n}^{0}(x)\right) \\
\geq-\left(\frac{1}{\lambda}\left[e^{\lambda k^{2}}-1\right]+\frac{1}{\lambda}\left[e^{4 \lambda k^{2}}-e^{\lambda k^{2}}\right]\right)|\Omega|-2 k e^{4 \lambda k^{2}}\left\|\left(u_{n}^{0}\right)^{-}\right\|_{L^{1}(\Omega)} .
\end{gathered}
$$

Thus, by (2.2), (2.14) and the assumptions on $f$ we deduce, since the function $\varphi_{\lambda}\left(T_{k}(s)-k\right) \leq 0, \forall s \in \mathbb{R}$,

$$
\begin{aligned}
\alpha \int_{Q_{\Omega}^{T}} \mid & \left.\nabla T_{k}\left(u_{n}\right)\right|^{p} \varphi_{\lambda}^{\prime}\left(T_{k}\left(u_{n}\right)-k\right)-\int_{Q_{\Omega}^{T}} \gamma_{k}\left|\nabla T_{k}\left(u_{n}\right)\right|^{p}\left|\varphi_{\lambda}\left(T_{k}\left(u_{n}\right)-k\right)\right| \\
\leq & \varphi_{\lambda}(2 k) \int_{Q_{\Omega}^{T}}\left[\left|f_{n}^{-}\right|+\left|g_{k}(t, x)\right|\right]+2 k e^{4 \lambda k^{2}}\left\|\left(u_{n}^{0}\right)^{-}\right\|_{L^{1}(\Omega)} \\
& +\left(\frac{1}{2 \lambda}\left[e^{\lambda k^{2}}-1\right]+\frac{1}{\lambda}\left[e^{4 \lambda k^{2}}-e^{\lambda k^{2}}\right]+2 k^{2} e^{4 \lambda k^{2}}\right)|\Omega|
\end{aligned}
$$

By fixing a suitable $\lambda>0$, so that (2.20) holds for $\varphi_{\lambda}(s)$, we deduce that $k-T_{k}\left(u_{n}\right)$ is bounded in $L^{p}\left(0, T ; W_{0}^{1, p}(\Omega)\right)$ and so, up to subsequences (not relabeled), it weakly converges in $L^{p}\left(0, T ; W_{0}^{1, p}(\Omega)\right)$.

As already pointed out, the conclusion of the Theorem follows exactly using the same steps of Theorem 2.3 


\section{Further Regularity}

In this section we are going to describe some local regularity properties for a renormalized solution of problem

$$
\begin{cases}u_{t}-\operatorname{div} a(t, x, u, \nabla u)+g(t, x, u, \nabla u)=f(t, x) & \text { in }(0, T) \times \Omega \\ u(0, x)=u_{0}(x) & \text { in } \Omega,\end{cases}
$$

where $\Omega$ is a, possibly unbounded, domain in $\mathbb{R}^{N}$.

Let us first emphasize that in this section we would like to be able to choose test functions of the type $S^{\prime}(u) \psi$ with $S^{\prime}$ not compactly supported on $\mathbb{R}$ and such that $\psi(T, x) \neq 0$. In principle, according to Definition 2.2 we are not allowed to do that. Anyway, after suitably modifying our definition, this fact can be made rigourous by an easy density argument. In fact, we can choose $S^{\prime}(u)=S_{j}^{\prime}(u) M(u)$ where $M$ is a Lipschitz function and $S_{j}$ is defined in (2.21), in the renormalized formulation. Then, we take the limit as $j$ diverges and we observe that $S_{j}^{\prime}(u)$ converges to 1 both a.e. and *-weak in $L^{\infty}\left(Q_{\Omega}^{T}\right)$, and the term involving $S^{\prime \prime}$ vanishes thanks to (2.11).

Moreover, we need to deal with cut-off functions which do not depend on time; to do that we choose a family of functions of the type $\phi_{\delta}(t, x)=\xi(x) \psi_{\delta}(t)$ such that $\xi(x) \in C_{0}^{1}(\Omega)$ and $\psi_{\delta}(t) \in C_{0}^{1}([0, T))$ that converges to $\chi_{[0, \tau]}$. Thus, according Proposition 2.6, a standard choice of $\psi_{\delta}(t)$ allows us to deduce the following equivalent formulation that is the useful one in order to obtain our regularity estimates:

$$
\begin{gathered}
\int_{\Omega} \mathcal{M}(u(\tau, x)) \xi(x)+\int_{Q_{\Omega}^{\tau}} a(t, x, u, \nabla u) \cdot \nabla u M^{\prime}(u) \xi \\
+\int_{Q_{\Omega}^{\tau}} a(t, x, u, \nabla u) \cdot \nabla \xi M(u) \\
+\int_{Q_{\Omega}^{\tau}} g(t, x, u, \nabla u) M(u) \xi=\int_{Q_{\Omega}^{\tau}} f(t, x) M(u) \xi+\int_{\Omega} \mathcal{M}\left(u_{0}\right) \xi(x),
\end{gathered}
$$

for any $0<\tau \leq T, \xi(x) \in C_{0}^{1}(\Omega)$, and $\mathcal{M}^{\prime}(s)=M(s)$, with $\mathcal{M}(0)=0$.

Finally, we observe that, since the estimates we are going to prove in this section are localized, we will proceed as follows. We fix a ball (without loss of generality, centered at 0 ) of radius $R$ contained in $\Omega$. Thus there exists $\rho>0$ such that $B_{R+\rho} \subset \subset \Omega$ and we will prove the estimates in $Q_{R}^{T}$, depending on quantities computed on $Q_{R+\rho}^{T}$. By covering any compact $\omega \subset \subset \Omega$ with balls we then obtain the results.

We start proving Proposition 2.6.

of Proposition 2.6. . According to the formulation (5.2) we are allowed to choose $\psi(t, x)=\xi(x)$, where $\xi$ is chosen as in (4.1) and such that Proposition 3.1 holds true, and $M(s)=T_{k}(s), \forall k \geq L$. Thus we have, recalling that $\Theta_{k}(s)=\int_{0}^{s} T_{k}(\tau) d \tau$

$$
\begin{gathered}
\int_{B_{R+\rho}} \Theta_{k}(u(x, t)) \xi^{p}+\frac{\alpha}{2^{p-1}} \int_{Q_{R+\rho}^{T}}\left|\nabla\left(T_{k}(u) \xi\right)\right|^{p}+\frac{1}{2} \int_{Q_{R+\rho}^{T}} h\left(|\nabla u|^{p-1}\right) T_{k}(u) \xi^{p} \\
\leq k\|f\|_{L^{1}\left(Q_{R+\rho}^{T}\right)}+C_{0}+\alpha \int_{Q_{R+\rho}^{T}}\left|T_{k}(u)\right|^{p}|\nabla \xi|^{p}+k\left\|u_{0}\right\|_{L^{1}\left(B_{R+\rho}\right)} \cdot
\end{gathered}
$$

Note that, since Proposition 3.8 holds true, then $u^{p-1}$ belongs to $L^{1}\left(Q_{R}^{T}\right)$ and so the last integral can be estimated by $C k$, for suitable $C>0$. Thus we deduce, by 
dropping positive terms,

$$
\int_{Q_{R}^{T}}\left|\nabla T_{k}(u)\right|^{p} \leq C(k+1)
$$

and so we conclude applying Lemma 3.9. Moreover the embedding of Marcinkiewicz spaces into Lebesgue ones (see (2.16) ) together with the assumption $p>2-\frac{1}{N+1}$ allow us to say that $u$ is bounded in $L^{r}\left(0, T ; W_{\text {loc }}^{1, r}(\Omega)\right)$ for some $r>1$, while from the equation in (5.1) (which is satisfied in the sense of distributions) we observe have $u_{t} \in L_{l o c}^{1}\left(Q^{T}\right)+L^{r^{\prime}}\left(0, T ; W_{l o c}^{-1, r^{\prime}}(\Omega)\right)$. Thus the continuity with values in $L_{\text {loc }}^{1}$ is an easy consequence of Theorem 1.1 of [27.

Finally we give an idea of the proof of Theorem 2.7.

Sketch of the proof of Theorem 2.7. We start by giving the proof of $(i)$ and (ii).

Let us fix any $0<R<R+\rho$ and consider $B_{R} \subset B_{R+\rho} \subset \subset \Omega$. Let us choose $M(s)=v_{\varepsilon, j}(s)$, and $\psi=\xi^{\lambda}$ in (5.2), where $\lambda=\max \left\{p, \frac{q^{\prime} p}{q^{\prime} p-1}, \frac{q^{\prime} p^{\prime}}{q^{\prime} p^{\prime}-1}\right\}, \xi(x)$ is as in (4.1) and

$$
v_{\varepsilon, j}(s)=\left[\left(\left|T_{j}(s)\right|+\varepsilon\right)^{\gamma}-\varepsilon^{\gamma}\right] \operatorname{sign} s,
$$

for any $0<\gamma \leq \bar{\gamma}$, with

$$
\bar{\gamma}= \begin{cases}\frac{N m(q-1)+q(m-1)[p(N+1)-2 N]}{N m-p q(m-1)} & \text { if (2.17) holds } \\ \frac{N(p-1)(q-1)}{N-p q} & \text { if (2.18) holds . }\end{cases}
$$

We follow the same ideas of previous estimates, using the ellipticity condition, assumption (2.7) and Proposition 3.1. and finally letting $\varepsilon$ tend to zero. Thus, we deduce that there exists a constant $C=C(\alpha, \beta, L, N, R, \rho, m, q)>0$, but independent of $j$, such that,

$$
\begin{gathered}
\left\|\xi^{\frac{\lambda(\gamma+p-1)}{p(\gamma+1)}} T_{j}(u)\right\|_{L^{\infty}\left(0, T ; L^{\gamma+1}\left(B_{R+\rho}\right)\right)}^{\gamma+1}+\int_{0}^{T}\left\|\xi^{\frac{\lambda}{p}}\left|T_{j}(u)\right|^{\frac{\gamma+p-1}{p}}\right\|_{L^{p^{*}}\left(B_{R+\rho}\right)}^{p} \\
\leq C\left[\|f\|_{L^{m}\left(0, T ; L^{q}\left(B_{R+\rho}\right)\right)}\left(\int_{0}^{T}\left(\int_{B_{R+\rho}} \xi^{\frac{\lambda}{p}}\left|T_{j}(u)\right|^{\gamma q^{\prime}}\right)^{\frac{m^{\prime}}{q^{\prime}}}\right)^{\frac{1}{m^{\prime}}}\right. \\
\left.+\int_{Q_{R+\rho}^{T}} \xi^{\lambda-p}\left|T_{j}(u)\right|^{p+\gamma-1}+\int_{B_{R+\rho}}\left|u_{0}\right|^{\gamma+1}+1\right],
\end{gathered}
$$

where we have applied a space-time Hölder inequality on the term involving the datum $f(t, x)$. Using the interpolation inequality with respect to the space variable and Young inequality with respect to the time variable, we deduce both that

$$
\left\|T_{j}(u)\right\|_{L^{\infty}\left(0, T ; L^{\gamma+1}\left(B_{R}\right)\right)} \leq C_{1}\left[1+\left\|T_{j}(u)\right\|_{L^{p+\gamma-1}\left(Q_{R+\rho}^{T}\right)}^{p+\gamma-1}\right]^{\frac{1}{\gamma+1}},
$$

and

$$
\left\|T_{j}(u)\right\|_{L^{\gamma+p-1}\left(0, T ; L^{\frac{\gamma+p-1}{p} p^{*}}\left(B_{R}\right)\right)} \leq C_{1}\left[1+\left\|T_{j}(u)\right\|_{L^{p+\gamma-1}\left(Q_{R+\rho}^{T}\right)}^{p+\gamma-1}\right]^{\frac{1}{\gamma+p-1}},
$$

where $C_{1}$ also depends on $\|f\|_{L^{m}\left(0, T ; L^{q}\left(B_{R+\rho}\right)\right)}$ and, since $\gamma \leq \bar{\gamma}$, on $\left\|u_{0}\right\|_{L^{\bar{\gamma}+1}\left(B_{R+\rho}\right)}^{\bar{\gamma}+1}$, too. Note that $\bar{\gamma}+1$ is the best summability we can expect as far as the initial 
datum is concerned. By (5.4), (5.5) and by applying inequality (3.11) to the function $\left|T_{j}(u)\right|^{\frac{\gamma+p-1}{p}}$, we have:

$$
\begin{aligned}
& \left\|T_{j}(u)\right\|_{L^{\frac{p+\gamma-1}{p} \eta}\left(Q_{R}^{T}\right)}^{\frac{p+\gamma-1}{p}} \\
& \leq C\left\|T_{j}(u)\right\|_{L^{\infty}\left(0, T ; L^{\gamma+1}\left(B_{R}\right)\right)}^{1-\theta}\left\|T_{j}(u)\right\|_{L^{\gamma+p-1}\left(0, T ; L^{\frac{\gamma+p-1}{p} p^{*}}\left(B_{R}\right)\right)}^{\theta},
\end{aligned}
$$

where $\eta$ and $\theta$ satisfy

$$
\frac{1}{\eta}=\frac{\theta}{p^{*}}+\frac{(1-\theta)(\gamma+p-1)}{p(\gamma+1)}, \quad \frac{p}{\theta} \geq \eta
$$

It is easy to see that, if

$$
\eta=p\left[1+\frac{p(\gamma+1)}{N(\gamma+p-1)}\right]
$$

then the above constraints are optimized. Thus gathering together (5.6), (5.4) and (5.5) we deduce that there exists $C>0$ such that

$$
\left\|T_{j}(u)\right\|_{L}{ }_{\frac{\eta(\gamma+p-1)}{p}\left(Q_{R}^{T}\right)} \leq C\left[1+\left\|T_{j}(u)\right\|_{L^{\gamma+p-1}\left(Q_{R+\rho}^{T}\right)}^{\gamma+p-1}\right]^{\frac{p}{\gamma+p-1} \max \left\{\frac{1}{\gamma+p-1}, \frac{1}{\gamma+1}\right\}} .
$$

Therefore, we control the norm of $T_{j}(u)$ in $L^{\frac{\eta(\gamma+p-1)}{p}}$ of a cylinder with the norm in $L^{\gamma+p-1}$ of a slightly larger cylinder. Moreover such estimate is uniform with respect to $j$. Noticing that $\eta>p$, in order to conclude it is enough to perform an iteration method. We can construct both $\bar{k}+1$ radii $0=\rho_{0}<\rho_{1}, \ldots, \rho_{\bar{k}-1}<\rho_{\bar{k}}=\rho$ and $\bar{k}+1$ exponents $\gamma_{0}<\gamma_{1}, \ldots, \gamma_{\bar{k}-1}<\gamma_{\bar{k}}=\bar{\gamma}$, such that

$$
\gamma_{0}+p-1<p-1+\frac{p}{N}
$$

and $\frac{\eta\left(\gamma_{\bar{k}}+p-1\right)}{p}$ is our desired summability.

Thus, applying (5.7) $\bar{k}+1$ times and using Proposition 2.6. we get the result. To deal with the different time-space summability stated in Theorem 2.7 we can argue in a similar way by applying Hölder inequality.

Now we deal with part (iii) of the Theorem. Let us denote by

$$
A_{k, r}=\left\{x \in B_{\rho}\left(x_{0}\right):|u(t, x)|>k\right\}, \forall r>0,
$$

and let us set, for any fixed $\delta \in(0,1)$,

$$
\begin{gathered}
t_{1}=\left[\frac{1-\delta}{\left|B_{R+\rho}\right|^{\lambda_{1}}}\right]^{\lambda_{2}} \\
\lambda_{1}=\left(1-\frac{\sigma}{q(\sigma-p)}\right)\left(1-\frac{p}{\sigma}\right) \quad \text { and } \quad \lambda_{2}=\frac{m \mu}{m(\mu-p)-\mu},
\end{gathered}
$$

where

$$
\sigma=p \frac{N m^{\prime}+p q^{\prime}}{N m^{\prime}}, \quad \mu=p \frac{N m^{\prime}+p q^{\prime}}{N q^{\prime}} .
$$

Let us choose $M(u)=T_{j}\left(G_{k}(u)\right)$ in (5.2), $j>k>L$ and $\psi=\xi^{p}$ ( $\xi$ chosen as in (4.1)) in the cylinder of height $t_{1}$, where we will fix $\delta$ (and so $t_{1}$ ) later. 
We also recall that for $\xi$ chosen as in (4.1), we have $|\nabla \xi| \leq \frac{c}{\rho}$. Thus, by standard computations, we have

$$
\begin{aligned}
& \int_{0}^{t_{1}} \int_{A_{k, R+\rho}}\left|\nabla T_{j}\left(G_{k}(u)\right)\right|^{p} \xi^{p} \leq \frac{c_{1}}{\rho} \int_{Q_{R}^{t_{1}}}\left|T_{j}\left(G_{k}(u)\right)\right| \xi^{p-1} \\
& +\int_{0}^{t_{1}} \int_{A_{k, R+\rho}}|f|\left|T_{j}\left(G_{k}(u)\right)\right|^{p} \xi^{p}+\int_{0}^{t_{1}} \int_{A_{k, R+\rho}}|f| \xi^{p} .
\end{aligned}
$$

Moreover, we choose $M_{\varepsilon}(u)=\left[\left(\left|T_{j}\left(G_{k}(u)\right)\right|+\varepsilon\right)^{p-1}-\varepsilon^{p-1}\right] \operatorname{sign} u, j>k>L$ and $\psi=\xi^{p}$ in (5.2). Thus, dropping positive terms, as $\varepsilon$ goes to zero, we get

$$
\begin{aligned}
\sup _{t \in\left(0, t_{1}\right)} & \int_{A_{k, R+\rho}} \Theta_{j}\left(\left|G_{k}(u)\right|^{p}\right)(t) \xi^{p} \leq \frac{c_{2}}{\rho} \int_{0}^{t_{1}} \int_{A_{k, R+\rho}}\left|T_{j}\left(G_{k}(u)\right)\right|^{p-1} \xi^{p-1} \\
& +c_{3} \int_{0}^{t_{1}} \int_{A_{k, R+\rho}}|f| \xi^{p}+c_{4} \int_{0}^{t_{1}} \int_{A_{k, R+\rho}}|f|\left|T_{j}\left(G_{k}(u)\right)\right|^{p} \xi^{p}
\end{aligned}
$$

where, as before, $\Theta_{j}(s)$ denotes the primitive of $T_{j}(s)$ such that $\Theta_{j}(0)=0$. Now we apply Corollary 3.7 with $w=T_{j}\left(\left|G_{k}(u)\right|\right) \xi, \Omega=B_{R+\rho}$ and $T=t_{1}$. Thus, for all $\mu$ and $\sigma$ satisfying (3.10), we deduce, by adding (5.11) and (5.12), and by applying Corollary 3.7

$$
\begin{aligned}
& {\left[\int_{0}^{t_{1}}\left(\int_{A_{k, R+\rho}}\left(\left|T_{j}\left(G_{k}(u)\right)\right| \xi\right)^{\sigma}\right)^{\frac{\mu}{\sigma}}\right]^{\frac{p}{\mu}} \leq \frac{c_{5}}{\rho^{p}} \int_{0}^{t_{1}} \int_{A_{k, R+\rho}}\left|T_{j}\left(G_{k}(u)\right)\right|^{p_{0}}} \\
& \quad+c_{6} \int_{0}^{t_{1}} \int_{A_{k, R+\rho}}|f|\left|T_{j}\left(G_{k}(u)\right)\right|^{p} \xi^{p}+c_{7} \int_{0}^{t_{1}} \int_{A_{k, R+\rho}}|f| \xi^{p},
\end{aligned}
$$

with $p_{0}=\max \{1, p-1\}$. Recalling the definitions of $\mu$ and $\sigma$ (see (5.10) ) and noticing that both of them are greater than $p$, we use Hölder inequality to estimate the right hand side of (5.13), so that

$$
\begin{gathered}
\int_{0}^{t_{1}} \int_{A_{k, R+\rho}}\left|f \| T_{j}\left(G_{k}(u)\right)\right|^{p} \xi^{p} \\
\leq\left|B_{R+\rho}\right|^{\lambda_{1}} t_{1}^{\frac{1}{\lambda_{2}}}\left\|\xi T_{j}(u)\right\|_{L^{\sigma}\left(0, t_{1} ; L^{\mu}\left(A_{k, R+\rho}\right)\right)}^{p}\|f\|_{L^{m}\left(0, t_{1} ; L^{q}\left(B_{R+\rho}\right)\right)},
\end{gathered}
$$

where $\lambda_{1}$ and $\lambda_{2}$ have been defined in (5.9). We fix now $\delta$ (and consequently we fix $\left.t_{1}\right)$ such that $c_{6}\|f\|(1-\delta)<\frac{1}{2}$; note that this quantity depends on the data of the problem but not on $u$. Thus from (5.13) we deduce

$$
\begin{gathered}
{\left[\int_{0}^{t_{1}}\left(\int_{A_{k, R+\rho}}\left|T_{j}\left(G_{k}(u)\right)\right|^{\sigma} \xi^{\sigma}\right)^{\frac{\mu}{\sigma}}\right]^{\frac{p}{\mu}}} \\
\leq \int_{0}^{t_{1}} \int_{A_{k, R+\rho}}|f| \xi^{p}+\frac{c}{\rho^{p}} \int_{0}^{t_{1}} \int_{A_{k, R+\rho}}\left|T_{j}\left(G_{k}(u)\right)\right|^{p_{0}} .
\end{gathered}
$$

Moreover, by Hölder inequality it follows that, for every $h>k$,

$$
\left[\int_{0}^{t_{1}}\left(\int_{A_{k, R+\rho}}\left|T_{j}\left(G_{k}(u)\right)\right|^{\sigma} \xi^{\sigma}\right)^{\frac{\mu}{\sigma}}\right]^{\frac{p}{\mu}} \geq(h-k)^{p}\left(\int_{0}^{t_{1}}\left|A_{h, R}\right|^{\frac{m^{\prime}}{q^{\prime}}}\right)^{\frac{p}{\mu}} .
$$


Now we estimate the right hand side of (5.14): we first note that

$$
\int_{0}^{t_{1}} \int_{A_{k, R+\rho}}|f| \xi^{p} \leq\|f\|_{L^{m}\left(0, t_{1} ; L^{q}\left(B_{R+\rho}\right)\right)}\left(\int_{0}^{t_{1}}\left|A_{k, R+\rho}\right|^{\frac{m^{\prime}}{q^{\prime}}}\right)^{\frac{1}{m^{\prime}}},
$$

and moreover

$$
\int_{0}^{t_{1}} \int_{A_{k, R}}\left|T_{j}\left(G_{k}(u)\right)\right|^{p_{0}} \leq c\left\|T_{j}\left(G_{k}(u)\right)\right\|_{L^{d p_{0}}\left(Q_{R+\rho}^{t_{1}}\right)}^{p_{0}}\left(\int_{0}^{t_{1}}\left|A_{k, R+\rho}\right|^{\frac{m^{\prime}}{q^{\prime}}}\right)^{\frac{1}{m^{\prime}}},
$$

where $d=\max \{q, m\}$. Now, we observe that $f(t, x) \in L^{m_{0}}\left(0, t_{1} ; L^{q_{0}}\left(B_{R+\rho}\right)\right)$, $\forall m_{0}, q_{0}$ such that $1<m_{0} \leq m, 1<q_{0} \leq q$. In particular we can choose $m_{0}, q_{0}$ such that

$$
\begin{gathered}
m_{0} q=m q_{0} \quad \text { and } \quad \frac{1}{m_{0}}+\frac{N}{p q_{0}}=1+\varepsilon, \\
\forall \varepsilon<\min \left\{\frac{m_{0} q_{0}(N+p)+N(p-2)\left(q_{0}\left(m_{0}-1\right)+m_{0}\right)}{p_{0} d}, \frac{N}{p m_{0}}\right\} .
\end{gathered}
$$

Using the first part of the Theorem we deduce that $u \in L^{\hat{s}}\left(Q_{R+\rho}^{t_{1}}\right)$, where $\hat{s}=$ $\frac{m_{0} q_{0}(N+p)+N(p-2)\left(q_{0}\left(m_{0}-1\right)+m_{0}\right)}{\varepsilon}$. Since $\hat{s} \geq p_{0} d$ we deduce

$$
\frac{c}{\rho} \int_{0}^{t_{1}} \int_{A_{k, R}}\left|T_{j}\left(G_{k}(u)\right)\right|^{p_{0}} \leq \frac{c_{1}\|u\|_{L^{\hat{s}}\left(Q_{R+\rho}^{t_{1}}\right)}}{\rho}\left(\int_{0}^{t_{1}}\left|A_{k, R+\rho}\right|^{\frac{m^{\prime}}{q^{\prime}}}\right)^{\frac{1}{m^{\prime}}} .
$$

Gathering together the above informations, we finally deduce, using also that $\frac{\mu}{\sigma}=$ $\frac{m^{\prime}}{p q^{\prime}}$ and passing to the limit with respect to $j$, that there exists $C>0$ such that

$$
\int_{0}^{t_{1}}\left|A_{h, R}\right|^{\frac{m^{\prime}}{q^{\prime}}} \leq \frac{c}{(h-k)^{\mu} \rho^{\frac{\mu}{p}}}\left(\int_{0}^{t_{1}}\left|A_{k, R+\rho}\right|^{\frac{m^{\prime}}{q^{\prime}}}\right)^{\frac{1}{m^{\prime}}} .
$$

Since (2.19) is in force, we have

$$
\frac{\mu}{m^{\prime} p}=\frac{1}{q^{\prime}}+\frac{p}{m^{\prime} N}=1+\frac{p}{N}-\frac{p}{N}\left(\frac{1}{m}+\frac{N}{p q}\right)>1,
$$

and so we can apply Lemma 3.10 to the function

$$
\zeta(h, d)=\int_{0}^{t_{1}}\left|A_{k, d}\right|^{\frac{m^{\prime}}{q^{\prime}}}(t) d t .
$$

Thus the proof is complete for $0 \leq t_{1}<T$. As already remarked, it is clear that the choice of $t_{1}$ only depends on the data of the problem and thus we can iterate and conclude the same estimate in the whole cylinder in a finite number of steps.

Acknowledgments. The authors are grateful to CMA-University of Oslo and to CMUC-Departamento de Matemática da Universidade de Coimbra for their kind hospitality.

\section{REFERENCES}

[1] Al Sayed, W., Veron, L.: On uniqueness of large solutions of nonlinear parabolic equations in nonsmooth domains, Adv. Nonlinear Stud. 9, 149-164 (2009).

[2] Bandle, C., Diaz, G., Diaz, J.I.: Solutions d'équations de réaction-diffusion non linéaires explosant au bord parabolique, C. R. Acad. Sci. Paris Sér. I Math. 318, 455-460 (1994).

[3] Bandle, C., Marcus, M.: Large solutions of semilinear elliptic equations: existence, uniqueness and asymptotic behavior, J. Anal. Math. 58, 9-24 (1992). 
[4] Bénilan, P., Boccardo, L., Gallouët, T., Gariepy, R., Pierre, M., Vazquez ,J. L.: An $L^{1}$-theory of existence and uniqueness of nonlinear elliptic equations, Ann. Scuola Norm. Sup. Pisa Cl. Sci. 22, 241-273 (1995).

[5] Blanchard, D., Murat, F.: Renormalised solutions of nonlinear parabolic problems with $L^{1}$ data: existence and uniqueness. Proc. Roy. Soc. Edinburgh Sect. A 127, 1137-1152 (1997).

[6] Boccardo, L., Gallouët, T.: Nonlinear elliptic and parabolic equations involving measure data, J. Funct. Anal. 87, 149-169 (1989).

[7] Boccardo, L., Gallouët, T., Vazquez, J.L.: Nonlinear elliptic equations in $\mathbb{R}^{N}$ without growth conditions on the data, J. Differential Equations 105, 334-363 (1993).

[8] Boccardo, L., Gallouët, T., Vazquez, J.L.: Solutions of nonlinear parabolic equations without growth restrictions on the data, Electron. J. Differential Equations 60, 1-20 (2001).

[9] Boccardo, L., Murat, F., Puel, J.P.: $L^{\infty}$ estimate for some nonlinear elliptic partial differential equations and application to an existence result, SIAM J. Math. Anal. 23, 326-333 (1992).

[10] Boccardo, L., Porzio, M.M., Primo, A.: Summability and existence results for nonlinear parabolic equations, Nonlinear Analysis 71, 978-990 (2009).

[11] Brezis, H.: Semilinear equations in $\mathbb{R}^{N}$ without condition at infinity, Appl. Math. Optim. 12, 271-282 (1984).

[12] Carrillo, J., Wittbold, P.: Uniqueness of Renormalized Solutions of Degenerate EllipticParabolic Problems, Journal of Differential Equations 156, 93-121 (1999).

[13] Dall'Aglio, A., Orsina, L.: Nonlinear parabolic equations with natural growth conditions and $L^{1}$ data, Nonlinear Analysis 27, 59-73 (1996).

[14] Droniou, J., Prignet, A.: Equivalence between entropy and renormalized solutions for parabolic equations with smooth measure data, NoDEA 14, 181-205 (2007).

[15] Droniou, J., Porretta, A., Prignet, A.: Parabolic capacity and soft measures for nonlinear equations, Potential Anal. 19, 99-161 (2003).

[16] Grenon, N.: Existence results for some quasilinear parabolic problems, Ann. Mat. Pura Appl. $165,281-313$ (1993).

[17] Grenon, N., Mercaldo, A.: Existence and regularity results for solutions to nonlinear parabolic equations, Adv. Differential Equations 10, 1007-1034 (2005).

[18] Keller, J.B.: On solutions of $\Delta u=f(u)$, Commun. Pure Appl. Math. 10, 503-510 (1957)

[19] Landes, R.: On the existence of weak solutions for quasilinear parabolic boundary value problems, Proc. Royal Soc. Edinburgh Sect. A 89, 217-237 (1981).

[20] Leoni, F.,: Nonlinear elliptic equations in $\mathbb{R}^{N}$ with "absorbing" zero order terms, Adv. Differential Equations 5, 681-722 (2000).

[21] Leoni, F., Pellacci, B.: Local Estimates and global existence for nonlinear parabolic equations with absorbing lower order terms J. Evol. Equ. 6, 113-144 (2006).

[22] Leonori, T.: Large solutions for a class of nonlinear elliptic equations with gradient terms, Adv. Nonlin. Stud. 7, 373-270 (2007).

[23] Leray, J., Lions, J. L.: Quelques résultats de Višik sur les problémes elliptiques semi-linéaires par les méthodes de Minty et Browder, Bull. Soc. Math. France 93, 97-107 (1956).

[24] Nirenberg, L.: On elliptic partial differential equations, Ann. Scuola Norm. Sup. Pisa 13, 116-162 (1959).

[25] Osserman, R.: On the inequality $\Delta u \geq f(u)$, Pacific J. Math. 7, 1641-1647 (1957).

[26] Petitta, F.: Renormalized solutions of nonlinear parabolic equations with general measure data, Ann. Mat. Pura Appl. 187, 563-604 (2008).

[27] Porretta, A.: Existence results for nonlinear parabolic equations via strong convergence of truncations, Ann. Mat. Pura ed Appl. 177, 143-172 (1999).

[28] Simon, J.: Compact sets in the space $L^{p}(0, T ; B)$, Ann. Mat. Pura Appl. 146, 65-96 (1987).

[29] Stampacchia, G.: Le problème de Dirichlet pour les équations elliptiques du seconde ordre à coefficientes discontinus, Ann. Inst. Fourier (Grenoble) 15, 189-258 (1965).

[30] Veron, L.: Semilinear elliptic equations with uniform blow-up on the boundary, J. Analyse Math. 59, 231-250 (1992). 
Tommaso Leonori, Departamento de Análisis Matemático, Universidad de Granada, 18071 Granada, SPAin

E-mail address: leonori@ugr.es

Francesco Petitta, Departamento de Análisis Matemático, Universitat de Valencia, C/ Dr. Moliner 50, 46100, Burjassot, Valencia, Spain

E-mail address: francesco.petitta@uv.es 